\title{
Runaway electron beam dynamics at low plasma density in DIII-D: energy distribution, current profile, and internal instability
}

\author{
A Lvovskiy ${ }^{1}$, C Paz-Soldan ${ }^{2}$, N W Eidietis ${ }^{2}$, P Aleynikov ${ }^{3}$, \\ M E Austin ${ }^{4}$ A Dal Molin ${ }^{5}$, Y Q Liu' ${ }^{2}$, R A Moyer ${ }^{6}$, \\ M Nocente ${ }^{5}$, D Shiraki ${ }^{7}$, L Giacomelli ${ }^{8}$, W W Heidbrink ${ }^{9}$, \\ E M Hollmann ${ }^{6}$, D Rigamonti ${ }^{8}$, D A Spong ${ }^{7}$, and M Tardocchi ${ }^{8}$ \\ ${ }^{1}$ Oak Ridge Associated Universities, Oak Ridge, TN, USA \\ ${ }^{2}$ General Atomics, San Diego, CA, USA \\ 3 Max-Planck-Institut für Plasmaphysik, Greifswald, Germany \\ ${ }^{4}$ University of Texas, Austin, TX, USA \\ ${ }^{5}$ Dipartimento di Fisica, Università di Milano-Bicocca, Milan, Italy \\ ${ }^{6}$ University of California San Diego, La Jolla, CA, USA \\ ${ }^{7}$ Oak Ridge National Laboratory, Oak Ridge, TN, USA \\ ${ }^{8}$ Institute for Plasma Science and Technology, CNR, Milan, Italy \\ ${ }^{9}$ University of California, Irvine, Irvine, CA, USA \\ E-mail: lvovskiya@fusion.gat.com
}

\begin{abstract}
.
Parameters of the post-disruption runaway electron (RE) beam in the low density background plasma achieved after deuterium injection are investigated in DIII-D. The spatially resolved RE energy distribution function is measured for the first time during the RE plateau stage by inverting hard X-ray bremsstrahlung spectra. It has maximum energy up to $20 \mathrm{MeV}$ and a non-monotonic feature at 5-6 MeV observed only in the core of the beam supporting the possibility of kinetic instabilities. Results of FokkerPlank modelling qualitatively support the formation of the non-monotonic distribution function. The RE current profile is reconstructed for the first time using the spatially resolved RE energy distribution. It is found to be more peaked than the pre-disruption plasma current, with higher internal inductance, suggesting preferential formation of REs in the core plasma or potentially a radially inward motion of REs. The accessed relatively low current (180 kA) RE beam is found to be MHD stable, likely due to its elevated safety factor profile. From this base stable equilibrium, an internal MHD instability is accessed by ramping up the current. The instability leads to a sawtoothlike relaxation of the RE current profile, but drives no RE loss. An internal kink mode proposed as a candidate instability is supported by results of MARS-F modelling. Electron cyclotron emission (ECE) spectrum measured during the low density RE plateau is found to be bifurcated, with a break point at $\approx 100 \mathrm{GHz}$, suggesting resonant absorption of the ECE at low frequencies.
\end{abstract}

Keywords: tokamak disruptions, runaway electrons, energy distribution, current profile, MHD instabilities, electron cyclotron emission 
Submitted to: Nucl. Fusion 


\section{Introduction}

Generation of a large post-disruption runaway electron (RE) current poses a serious threat for present and future high-current tokamaks. Localized loss of a RE beam with energy up to tens of $\mathrm{MeV}$ and a current up to about ten MA can lead to severe melting and damage of plasma facing components and underlying structures in ITER [1,2]. Avoidance of RE generation or fast dissipation of rising RE current is considered as a preferable mitigation scheme for ITER [3]. For example, this could be done via prediction of a major tokamak disruption and timely injection of a large impurity amount to significantly increase the plasma collisionality before the RE acceleration and multiplication take place during the current quench (CQ). Feasibility and reliability of such a mitigation technique is yet to be proven [3]. Therefore it is important to assume that a strong RE beam can be generated in ITER and to develop a mitigation scenario for this case as a second line of defense. This requires detailed investigation into the physics of the post-disruption RE beam $[4,5]$. Significant research focus in the last years was made on the RE beam control [6-9] and the RE beam dissipation via secondary impurity injection [10-16] and resonant magnetic perturbations [17-20].

In this paper we address the equilibrium structure and magnetohydrodynamic (MHD) instabilities of the post-disruption RE beam at low plasma density in DIII-D. Observations of RE beam kinetic instabilities are presented separately in Ref. [21]. These experimental studies are generally difficult due to poor diagnosis of the internal structure of the RE beam. For example, evaluating the MHD stability of the RE beam requires evaluation of the internal current profile, a measurement that has until now been elusive. Evaluation of the kinetic stability requires evaluation of the distribution function, another elusive measurement during the intense RE plateau. These issues were recently overcome through implementation of novel high-flux bremsstrahlung measurements and operation of RE beams in low density low-Z background plasma. Using these advances we present measurements of the RE beam energy distribution function in Section 3 and reconstruction of the RE beam current profile in Section 4. Observation and investigation of an internal MHD instability leading to sawtooth-like redistribution of the RE current profile but no observable RE loss are given in Section 5. Observations of a bifurcated electron cyclotron emission spectrum from REs, made possible due to the low plasma density, are given in Appendix A. Validity of hard X-ray bremsstrahlung measurements during the RE plateau is discussed in Appendix B.

\section{Experimental setup}

Disruptions that generate RE beams are reliably triggered in DIII-D using argon killer pellet injection $[22,23]$ as shown in Figure 1. Due to significant amount of introduced argon (a single pellet contains $5 \cdot 10^{20}$ atoms, which is 2 times more particles than the target plasma), the post-disruption plasma is dense and has a large number of the high-Z impurity. In this work we assess the RE energy and spatial distribution 

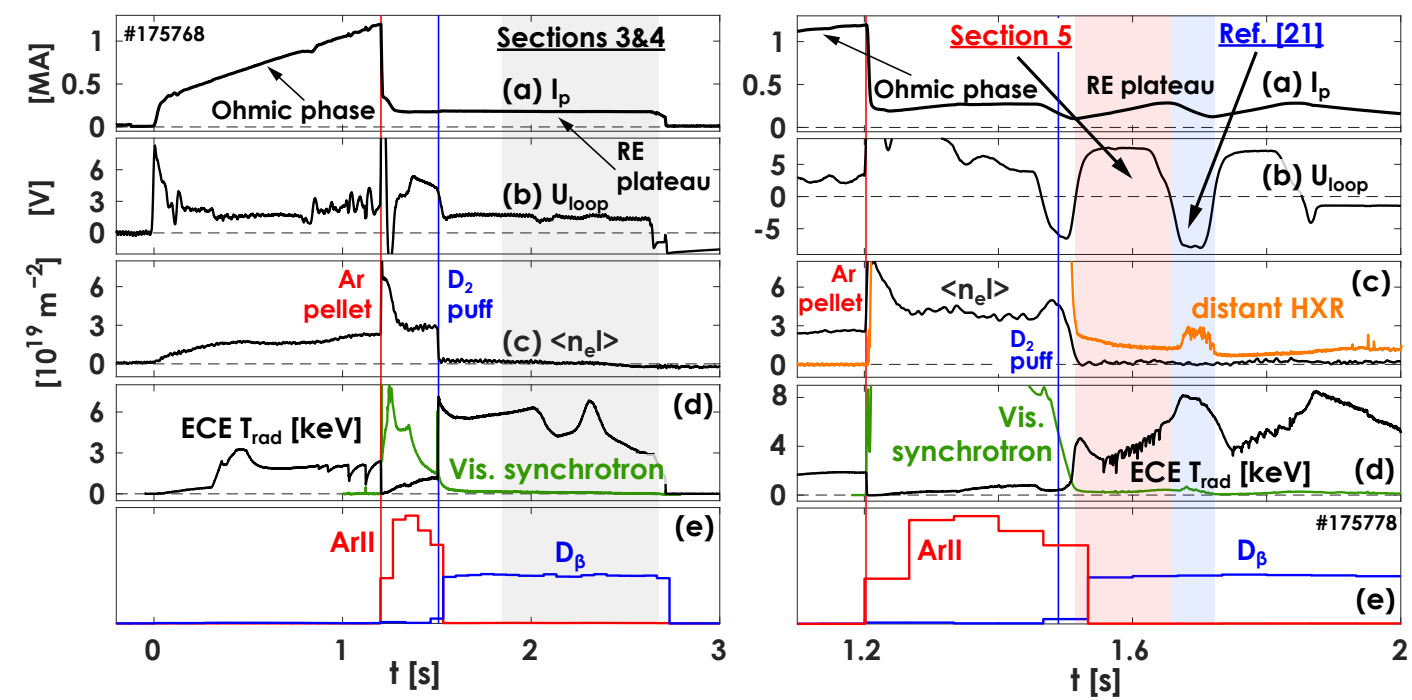

Figure 1: Time traces of (a) plasma current $\mathrm{I}_{p}$, (b) loop voltage $\mathrm{U}_{\text {loop }}$, (c) equatorial line integrated plasma density $\left\langle n_{e} l\right\rangle$ and (c-right) HXR signal both from confined and lost REs by the distant detector, (d) electron cyclotron emission at $113.5 \mathrm{GHz}$ and visible synchrotron emission at $790 \mathrm{~nm}$, (e) ArII spectral line $484.8 \mathrm{~nm}$ and $\mathrm{D}_{\beta}$ spectral line $486.1 \mathrm{~nm}$. Plasma current during the RE plateau stage is almost entirely carried by REs.

using measurements of the hard X-ray (HXR) bremsstrahlung radiation from confined REs (Sections 3 and 4). Dense plasma and abundant high-Z impurity prohibit these measurements since the power of bremsstrahlung radiation increases as $n_{e} Z^{2}$. To reduce the bremsstrahlung flux to a measurable level, the argon impurity is purged from the post-disruption plasma by a secondary injection of deuterium gas $\left(\sim 10^{22}\right.$ molecules $)$ as shown in Figure 1(c,e). Besides decreasing the amount of the high-Z impurity, this also decreases the electron density (measured by a $\mathrm{CO}_{2}$-interferometer) nearly to the noise level and provides the low-collisionality low-resistivity post-disruption background plasma. This effect was firstly observed in Ref. [15], and though it is not yet understood, it is hypothesized to be due to complete recombination of the background plasma.

Additionally to being better diagnosed in the HXR range, the low-resistivity plasma allows a long-lasting RE plateau ( $>1 \mathrm{~s}$ ) and a larger variability of applied loop voltage due to more effective utilization of transformer's flux. In the series of discharges the loop voltage was modified (by ramping the Ohmic solenoid) to large positive and negative values as shown in Figure 1(right) to study the MHD and kinetic stability of the RE beam. Their analysis is presented in Section 5 and Ref. [21] accordingly. 
Table 1: The list of maximum RE energy measured in tokamaks during the RE plateau stage.

\begin{tabular}{llll}
\hline Tokamak & Max $\mathbf{E}_{\mathbf{R E}}[\mathbf{M e V}]$ & How diagnosed & Refs. \\
\hline JET & 20 & Vertical HXR spectrometer & {$[12,30]$} \\
DIII-D & $20-60$ & Visible synchrotron camera & {$[28]$} \\
ASDEX-U & 10 and 25 & Neutron and radial HXR spectr. & {$[29,31]$} \\
TEXTOR & $25-30$ & IR sync. camera, scint. probe & {$[32-34]$} \\
JT-60U & $>26$ & Neutron monitor & {$[35]$} \\
Tore Supra & $10-15$ & Neutron monitor & {$[8]$} \\
TFTR & 13 & Tangential HXR spectrometer & {$[36]$} \\
\hline
\end{tabular}

\section{Energy distribution function of the RE beam}

\subsection{Motivation}

The energy distribution function is one of the most important characteristics of the RE beam. It provides information about maximum RE energy and major current carriers [2]. The shape of the distribution function can be used to estimate actuators accelerating and dissipating the RE beam [24,25]. Local features of the distribution function such as a 'bump-on-tail' are important in the analysis of the beam kinetic stability [26, 27]. Comparison of measured and theoretically calculated distribution functions is a way to verify models predicting physics of REs in ITER [24].

Experimental measurements of the RE beam energy distribution function are challenging. A broad range of RE energy and extreme emission fluxes significantly complicate the diagnosis. In many experiments only the maximum RE energy was estimated as shown in Table 1. Measurements of trace-level RE populations in lowdensity Ohmic plasma discharges in DIII-D was an important achievement but require model interpretation to be extrapolated to post-disruption conditions [24,25]. Attempts to reconstruct the RE distribution function during the RE plateau stage in DIII-D based on measurements by a combination of various diagnostics lacked information in the range of $0.1-10 \mathrm{MeV}$ [28]. The RE beam energy distribution function measured on ASDEX-U using the HXR bremsstrahlung radiation mostly provided volumetric and perpendicular to the RE beam movement measurements due to its radially-oriented collimated line of sight [29]. As such, no well constrained measurement of the postdisruption RE distribution function for the most important energies and pitch angles is found in the literature. Here we present the first such measurement.

In this section we diagnose the RE beam energy distribution function using the HXR bremsstrahlung radiation from parallel-moving confined REs via a tangential line of sight in DIII-D. Conditions and limitations of these measurements are described in detail below. 


\subsection{Details of measurements}

Measurements of the RE energy distribution function are performed during the RE plateau stage at a relatively small $180 \mathrm{kA}$ RE current in the low-collisionality background plasma with a trace amount of high-Z species (Figure 1(left)). These conditions provide a long-lasting RE beam and reduce the HXR bremsstrahlung flux produced by REs via scattering on the background plasma which scales as $n_{e} Z^{2}$. Measurements of the bremsstrahlung radiation by the Gamma Ray Imager (GRI) allow construction of the HXR spectra via pulse height analysis (PHA) [37] and following inversion to the RE energy distribution function [24,25]. GRI is a novel diagnostic presenting a pin-hole camera with a 2D-array of gamma detectors imaging the poloidal cross-section of DIII$\mathrm{D}[37,38]$. Its lead collimation provides attenuation of non-collimated gammas by $95 \%$. HXR measurements during the RE plateau regime are challenging even at a small RE current and a small high-Z impurity content due to still very high bremsstrahlung flux and saturation of conventional GRI gamma detectors based on BGO crystals. To overcome this limitation, a novel ultrafast gamma detector consisting of a LYSO crystal $\left(\mathrm{Lu}_{1.8} \mathrm{Y}_{0.2} \mathrm{SiO}_{5}\right.$ :Ce) coupled to a Multi-Pixel Photon Counter has been developed and implemented to the GRI central line of sight [29,39-42]. This allowed increasing the time resolution by a factor of 1000x compared to the BGO detectors [37] and achieving $\mathrm{MHz}$ counting capabilities (discussion of the pile-up effect at the high counting rate can be found in Appendix B). HXR measurements are analyzed in $400 \mathrm{~ms}$ after the argon purge, during the last $800 \mathrm{~ms}$ of the RE plateau as shown in Figure 1(a-left).

\subsection{Results}

The measured HXR spectra and inverted RE spectra calculated for every $20 \mathrm{~ms}$ in the time range 1.9-2.7 s are shown in Figure 2(b,c). The time traces of plasma current, toroidal electric field and line-integrated plasma density are given in Figure 2(a). It can be seen that the RE energy spectrum is stationary during the time period 1.9$2.35 \mathrm{~s}$ (change of the ECE signal observed at that time in Figure 1(d) we explain by the small movement of the RE beam with respect to the mid-plane horizontal view of the ECE radiometer [43] as can be seen in Figure 4(a,e)). The following shift of the RE spectra to lower energies is caused by the vertical movement of the RE beam which displaces the beam with respect to the GRI sight line. This provides the vertical scan of the beam profile and is discussed in Section 4. The RE spectrum in the core extends up to $14 \mathrm{MeV}$ and has a non-monotonic feature (bump) at approximately 5-6 $\mathrm{MeV}$ in contrast to the monotonically decreasing energy distribution function expected from the classical Dreicer model with an avalanche term $[44,45]$. Peaking of the energy distribution function at low energy $(<100$ s of $\mathrm{keV})$ corresponding to the bulk electron population is not captured in the present experiment due to the noise threshold of the HXR measurements. The bump disappears and the maximum RE energy decreases down to $4 \mathrm{MeV}$ towards the beam edge. To obtain the temporally resolved spectra with a low noise, the threshold level is limited to $500 \mathrm{\gamma} / \mathrm{s}$ per energy bin. If the integration 


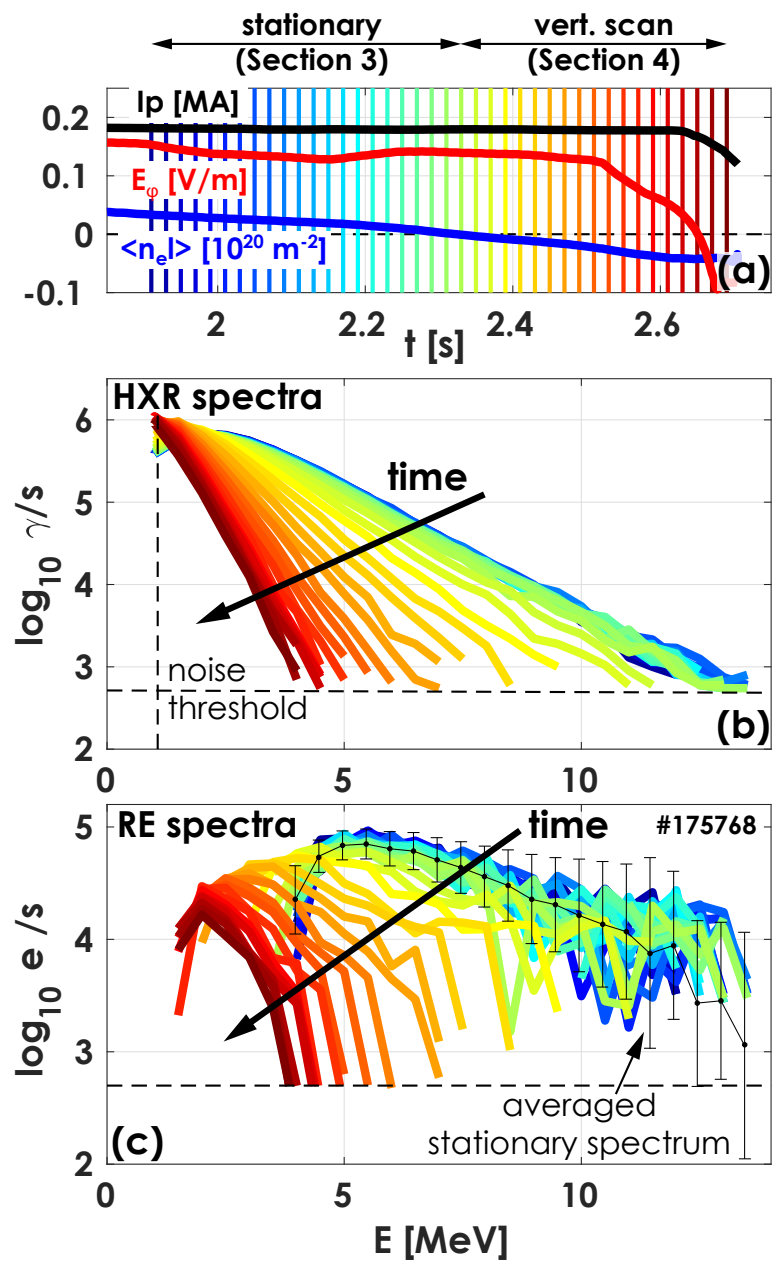

Figure 2: (a) Time traces of plasma current $\mathrm{I}_{p}$, toroidal electric field $E_{\phi}$, line-integrated plasma density $\left\langle n_{e} l\right\rangle$; (b) HXR and (c) RE spectra measured by the GRI with uncertainties based on Poisson counting-statistics [25]. Time slices are color-coded. Since the baseline of measurements by the $\mathrm{CO}_{2}$-interferometer can be affected by the changing geometry of the vacuum vessel and the plasma density is near the noise level, density values can be observed below the baseline after $2.4 \mathrm{~s}$ when the RE beam is moved vertically. The tail of the averaged stationary $R E$ spectrum is below the visible bins because some of initial RE spectra have no corresponding bins above the noise threshold.

time is increased and the threshold is softened, then HXRs corresponding to REs with maximum energy up to $20 \mathrm{MeV}$ can be resolved in the core (Figure 3(left)).

\subsection{Discussion}

The diagnosed energy distribution function of the RE beam has a maximum energy of $20 \mathrm{MeV}$ comparable with the maximum RE energy of 20-30 MeV typically observed in DIII-D and other tokamaks (Table 1). 
Conservation of the non-monotonic RE spectrum over $450 \mathrm{~ms}$ indicates that acceleration of REs by the toroidal electric field of $0.1-0.2 \mathrm{~V} / \mathrm{m}$ is likely balancing against RE dissipating factors which can be estimated as follows.

It is convenient to characterize the collisional damping of REs by the ratio of the toroidal electric field to the critical electric field required to observe the runaway [46]: $E / E_{c}=4 \pi \varepsilon_{0}^{2} m_{e} c^{2} E /\left(n_{e} e^{3} \ln \Lambda\right) \approx 10 \mathrm{U}_{\text {loop }}[\mathrm{V}] / n_{e}\left[10^{19} \mathrm{~m}^{-3}\right]$, where $\varepsilon_{0}$ is the vacuum electric permittivity, $m_{e}$ is the electron mass, $\ln \Lambda$ is Coulomb logarithm, $n_{e}$ is the electron density. If only thermal electrons are taken into account, the collisional damping is very small for given low density plasma: $E / E_{c}=40$ to 60 , while the characteristic time of RE energy loss via collisions [46] is very large: $\tau_{\text {coll }}=4 \pi \epsilon_{0}^{2} m^{2} c^{3} /\left(e^{4} n_{e} \ln \Lambda\right)=300$ to $600 \mathrm{~ms}$. This would lead to acceleration of REs to many tens of MeV. Thus it is important to include bound electrons into consideration (similar to Ref. [47]), which in this case are mostly provided by neutral deuterium. This accounting reduces $E / E_{c}$ ratio to a reasonable $1-1.5$ and $\tau_{\text {coll }}$ to $7 \mathrm{~ms}$. The significant role of neutral deuterium on dissipation of the RE current can be directly seen in discharges where the amount of injected deuterium is scanned. When the deuterium amount increases from $5.5 \cdot 10^{21}$ to $1.8 \cdot 10^{22}$ molecules, the loop voltage required to sustain the RE current rises from 0.55 to $1.6 \mathrm{~V}$.

The energy damping via synchrotron radiation is small for these plasma parameters. Its characteristic time $\tau_{\text {rad }}=6 \pi \varepsilon_{0} m_{e}^{3} c^{3} /\left(e^{4} B^{2}\right)$, where $B$ is the toroidal magnetic field, is about $160 \tau_{\text {coll }}$. The minimum time required for formation of the bump due to the synchrotron radiation [48,49]: $\tau_{\text {bump }} \approx 107\left(E / E_{c}-1\right) /\left(B^{2} \ln \Lambda\left(Z_{\text {eff }}+1\right)\right) \approx 350$ to 500 $\mathrm{ms}$, which is too large to explain the observation of the bump throughout the stationary spectrum. $Z_{\text {eff }}$ used above is the effective charge as defined in Ref. [47], and not to be confused with a usual thermal plasma $Z_{\text {eff }}$.

Considering other damping factors, we can exclude the effect of the bremsstrahlung radiation since it is small even compared to the weak synchrotron damping [50]; the orbit shift effect does not exceed a few $\mathrm{cm}$ at such a moderate energy of REs [51]; magnetic field ripple [52] or RE kinetic instabilities [53] need an additional treatment but are unlikely to be dominant factors setting the distribution.

It is interesting to note, that despite the conditions of the RE experiments carried out in the post-disruption plasmas and the low-density Ohmic flattop plasmas (also known as 'QRE regime' [54]) are different, both experiments show similar bumps at 5-6 MeV and 7-8 MeV respectively [24,25]. Though these non-monotonic features can be addressed to some instrumental effect or insufficient collimation, we do not consider these as the most likely explanations as discussed in Appendix B. Formation of the bump in the QRE experiments has been attributed to the interplay between electric field acceleration, collisional and synchrotron damping, and pitch-angle scattering [24, 25]. Later an important role of kinetic instabilities possibly altering the RE distribution function has been reported in Ref. [53]. 

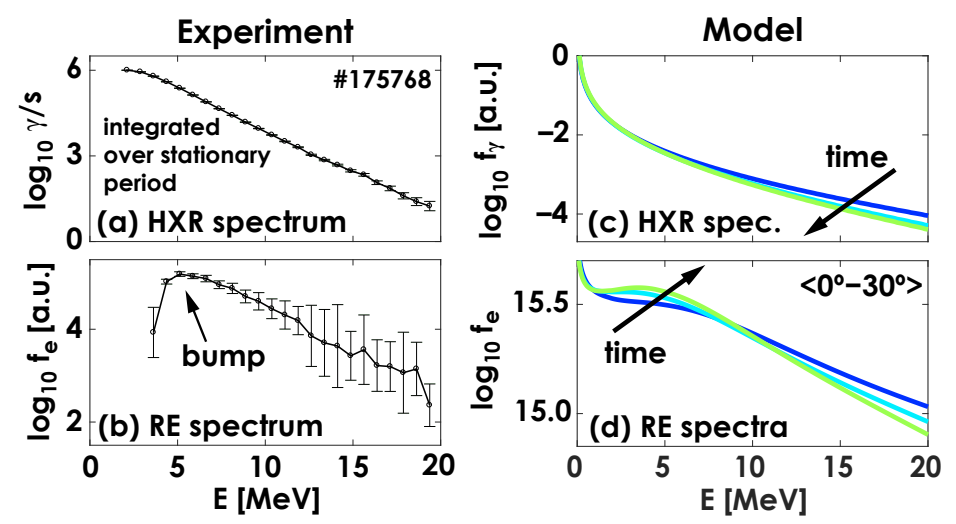

Figure 3: (a) Measured HXR and (b) invertion to RE spectra. (c) Forward modelled HXR and (d) modelled RE spectra integrated over pitch-angles of $0^{\circ}-30^{\circ}$. Time slices are color-coded same as in Figure 2. The vertical axis of (d) is expanded. The low-energy bulk electron population is not resolved in the experiment due to the noise threshold of HXR measurements shown in Figure 2(b).

\subsection{Fokker-Plank modelling}

Simple estimates show that formation of the stationary RE distribution function with a bump can be explained by the balance between acceleration under applied electric field and damping mostly via collisions with bound electrons.

To verify these results, the RE energy distribution function is modelled using the solution of the time-dependent relativistic Fokker-Plank equation with $E / E_{c}, \tau_{\text {rad }} / \tau_{\text {coll }}$, $Z_{\text {eff }}$ as input parameters. The equation from Ref. [55] is treated with a collision operator valid in the near-threshold regime and a secondary source taking into account the finite energy of interacting REs. Then the HXR distribution function is obtained from the calculated RE distribution via forward modelling as described in Refs. [24,25] with a pitch-angle integrated from $0^{\circ}$ to $30^{\circ}$ to be consistent with the angular measurement range of the GRI.

The modelled RE distribution function is shown in Figure 3(d). It captures the main features observed in the experiment such as the bump at approximately $5 \mathrm{MeV}$. The modelled bump slowly grows while the maximum energy slowly decreases over the simulated period, which is presumably explained by poorly diagnosed and overestimated damping factors.

\subsection{Conclusion}

The energy distribution function of the RE beam obtained via HXR measurements is found quasi-stationary in the low density plasma with a non-monotonic feature at 5-6 $\mathrm{MeV}$ suggesting the presence of free energy to drive kinetic instabilities. Results of the 0D-2V Fokker-Plank modelling reasonably match the observations when the collisional damping on bound electrons supplied by the neutral deuterium is taken into account. 


\section{Current profile of the RE beam}

\subsection{Motivation}

On existing tokamaks, only a small part of the pre-disruption plasma current is converted into the RE current. However, it was shown that the RE current profile is expected to be much more peaked than the pre-disruption current based on the modelling of the plasma current evolution during the disruption [56-58]. This is explained by significant narrowing of the current channel and increase of on-axis RE current even in excess of pre-disruption values. According to simulations [59-62], a peaked current profile can affect the stability of the RE beam and lead to the excitation of low-order MHD instabilities such as resistive kink and ideal internal kink. A different result is reported in Ref. [63], where sawtooth oscillations are claimed to be suppressed in the runaway plasma due to the flattening of the current profile after a single resistive internal kink mode.

Peaking of the RE current profile has been observed experimentally on JET using the soft X-ray emission to constrain the current density profile and calculate the safety factor $q$ profile [64]. Same result has been shown via estimations of the internal inductance $l_{i}(3)$ based on the plasma equilibrium reconstruction [65]. External kink instabilities leading to large and eventually complete RE loss have been recently studied for a high-current (1 MA) RE beam in DIII-D [66].

In this section we directly measure the RE current profile by taking moments of

the spatially resolved RE energy distribution function and evaluate its stability via equilibrium reconstruction with a constrained current density profile.

\subsection{Details of measurements}

As mentioned in Subsection 3.3, change of the RE spectra seen in Figure 2(c) after $t=2.35 \mathrm{~s}$ and until the final RE loss is caused by the vertical movement of the RE beam with respect to the GRI sight line, Figure 4(e). Since the minor radius of the beam stays nearly constant during this movement compared to the spectral change, Figure 4(c), the RE energy distribution functions, resolved with respect of time, Figure 2(c), can be mapped to the beam radial coordinate using the distance between the RE beam current centroid and the GRI sight line, which is also resolved with respect of time, Figure 4(d). As a result, the radial distribution of the RE current density can be found by taking the moments of the RE energy distribution functions.

The coordinates of the RE current centroid, minor radius, and the last closed flux surfaces (LCFSs) shown in Figure 4 are reconstructed using the JFIT code [67]. JFIT calculates the plasma current distribution using constraints by external magnetic measurements and fitting the distributed-current elements. This provides robust reconstruction of the plasma current during a disruption and a post-disruption stage. Validity of the reconstructed geometry is verified based on agreement of JFIT-based fits with measurements [67] and simulations [68] for halo current dynamics. Commonly 

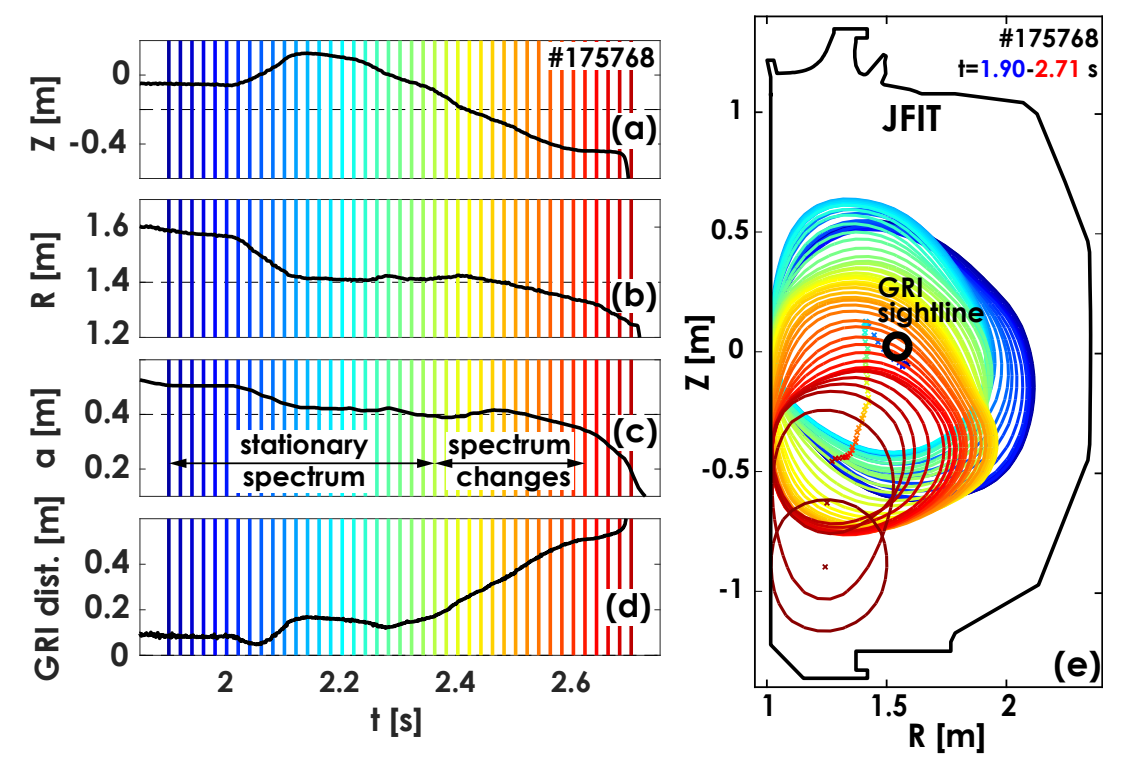

Figure 4: Time traces of (a) Z and (b) R coordinates of the RE beam current centroid, (c) minor radius $a$ of the $\mathrm{RE}$ beam, (d) distance between the current centroid and the GRI sight line, (e) LCFS contours. All values are calculated by JFIT. Accuracy of reconstructions is within a few $\mathrm{cm}$. The same color coding of time slices is used throughout Figures 2-4.

used magnetic equilibrium reconstruction by EFIT [69], applying solution of the GradShafranov equation as the constraint, often does not converge for typical disrupted plasmas in DIII-D due to low signals from the low current equilibria shifted away from the magnetic axis.

Figure 5(a) shows the raw RE current density profile measured by the GRI during the vertical scan. It is calculated from the radially mapped energy distribution functions via integration of the area under the curve. It can be seen that the current profile has a tail outside the LCFS. This feature can be reasonably explained by the nature of the bremsstrahlung radiation. The HXR bremsstrahlung is emitted in beam-forwarded cones based on the RE energy (Figure 5(b)). The lead collimator of the GRI reduces the uncollimated gamma-flux by $95 \%$ but HXRs passed through it can still be measured above the noise threshold level due to gamma fluxes exceeding $\mathrm{MHz}$ counting rate during the RE beam stage. Parameters of the HXR spectrum measured outside the LCFS support the hypothesis of an insufficient collimation: the gamma flux changes slowly (Figure B1(b), $t>2.55 \mathrm{~s}$ ) and has the maximum energy of 4-5 MeV (Figure 5(a)). This is in agreement with the lowest attenuation of $4 \mathrm{MeV}$ gammas by lead [70] and locally largest bremsstrahlung cross-section of $4 \mathrm{MeV}$ gammas at the angles $8^{\circ}-20^{\circ}$ (Figure 5(b)). Simple estimate shows that a mono-energetic $4 \mathrm{MeV}$ RE beam with a radius of $40 \mathrm{~cm}$ would be seen as twice bigger at the distance $140 \mathrm{~cm}$ (roughly the tangent distance between the GRI detector and the DIII-D magnetic axis).

To confirm that the RE current profile measured by the GRI outside the LCFS is an 

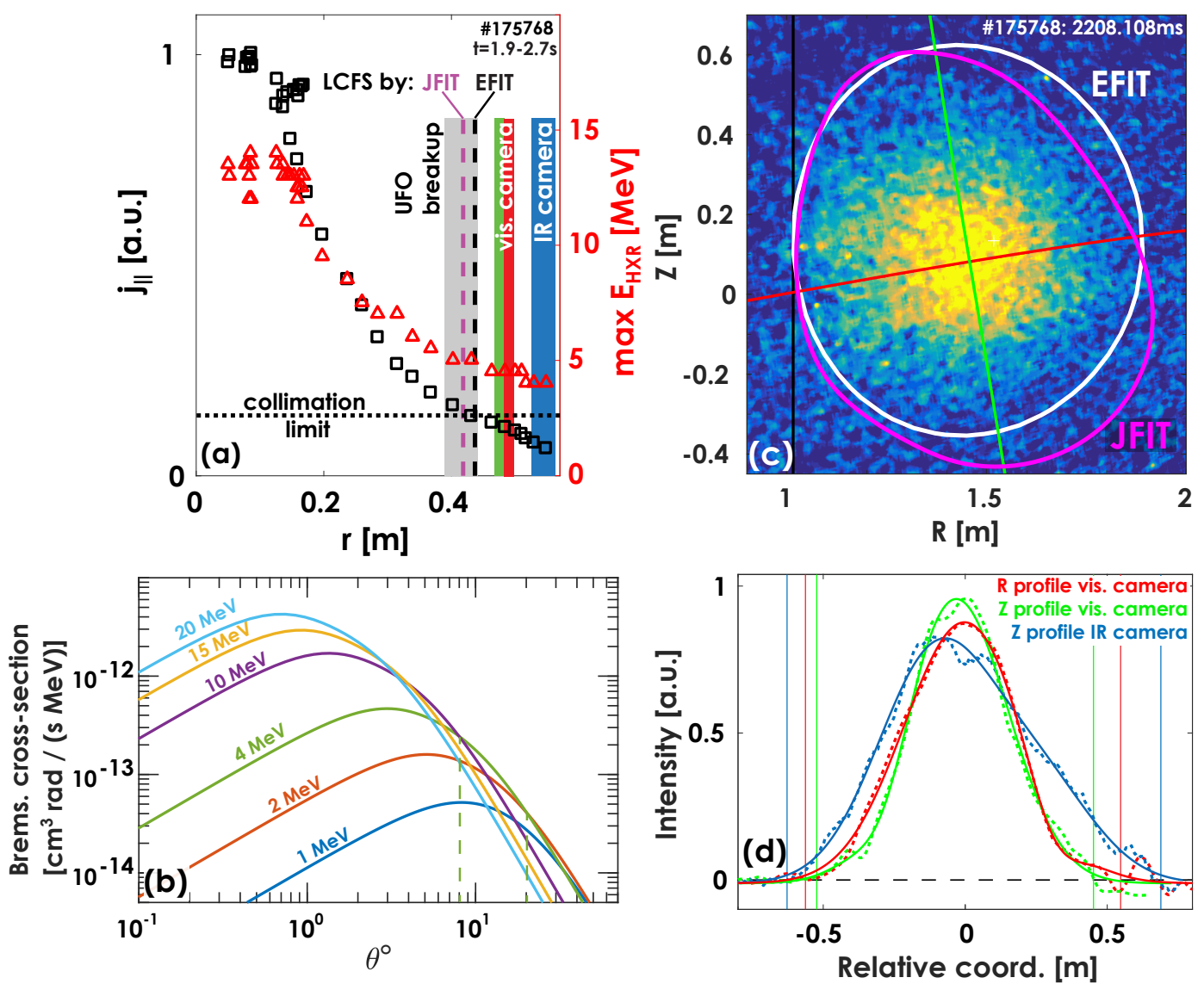

Figure 5: (a) (black) Raw RE current density profile and (red) maximum HXR energy measured by the GRI, (b) dependence of bremsstrahlung cross-section on RE energy, (c) RE beam imaged by the visible synchrotron camera, (d) Z and R emission intensity profiles of RE beam by the visible and IR cameras. LCFSs by JFIT and EFIT in (c) have a discrepancy probably because of a more coarse grid used in JFIT or different underlying constraints.

artifact of the HXR measurements and to assure that the LCFS reconstructed by JFIT indeed corresponds to the RE beam minor radius (since the RE beam could be located within some internal closed magnetic surfaces), we additionally consider measurements of the beam emission by visible synchrotron and infrared (IR) cameras (Figure 5(c,d)). Likely the most accurate estimate is provided via observations of bright microscopic particles of mobilized dust (so-called 'UFOs' [71]): a few times cameras recorded the disintegration of an UFO presumably happened when it hits the RE beam. As seen in Figure 5(a), the RE beam radii by the JFIT reconstruction and the UFO breakup well match to each other while the radii obtained from the emission profiles are slightly bigger. This discrepancy can be explained by the emission reflected from the wall which is difficult to subtract completely. The radius estimate provided by EFIT is also shown in Figure 5(a). According to our experience, EFIT can be not accurate in reconstruction of the RE current centroid but is able to constrain the LCFS when it converges. Based 


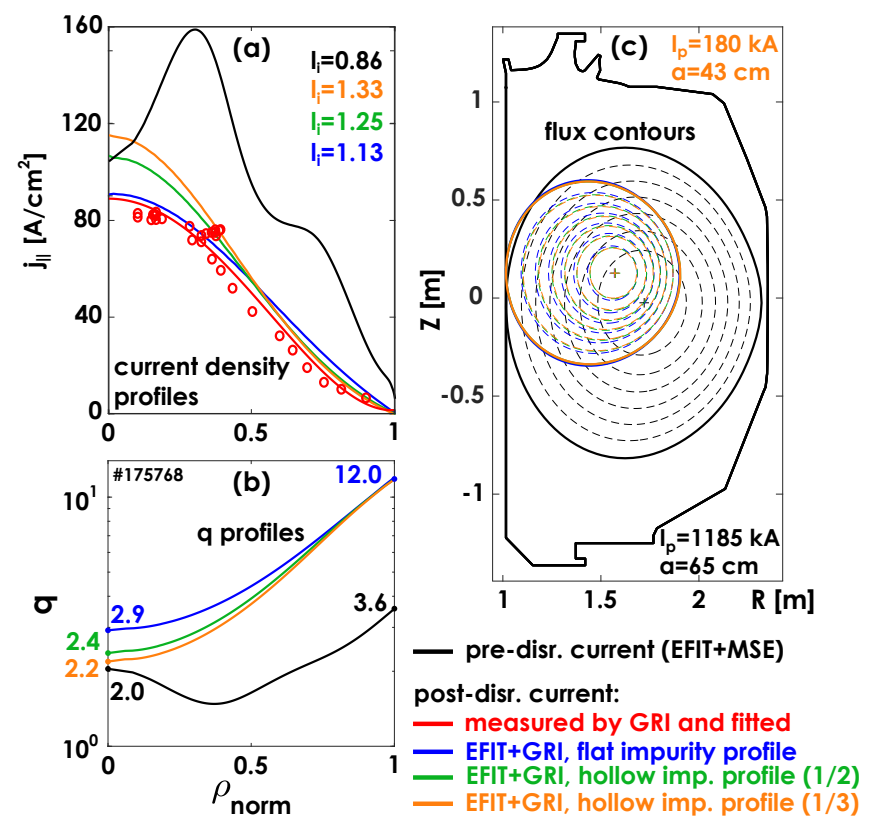

Figure 6: (a) Current density profiles of the RE beam and the pre-disruption plasma, (b) $q$ profiles, (c) EFIT flux contours.

on all these observation the radius of the RE beam is taken as $43 \mathrm{~cm}$. The current density outside of it is considered as the collimation limit and subtracted.

Since the GRI provides measurements along the tangential line of sight, the toroidal localization of the bremsstrahlung radiation is also important for reconstruction of the current profile. Considering the radiation from high-energy REs (above $5 \mathrm{MeV}$ ), the total HXR spectrum is localized within a relatively small region about $10 \mathrm{~cm}$ along the line of sight [37]. An effect of pitch-angle scattering of REs to large angles potentially reducing the toroidal localization of measurements can be ignored for given low-Z background plasma [28].

Finally, an additional treatment is needed to account for the impurity profile since the power of bremsstrahlung radiation depends on $n Z^{2}$. Based on the vanishing emission of argon spectral lines and the very small thermal electron density, we assume that the bremsstrahlung radiation is dominantly produced via scattering on neutral deuterium, thus only dependence on its density has to be considered. Since the density profile of neutral deuterium is poorly diagnosed, it is estimated taken the pressure profile flat, while the temperature increases towards the beam center, leading to a hollow density profile. Cases with a flat temperature profile and parabolic profiles increasing by factors of 2 and 3 compared to the wall temperature are considered. As shown below, the RE current profile is peaked even for the flat impurity profile.

\subsection{Results and discussion}

The toroidal RE current density profile measured by the GRI and normalized to the full RE current is shown in Figure 6(a). It is in a good agreement with the 
equilibrium reconstruction by EFIT using the GRI measurements as constraints. Similar reconstructions assuming hollow impurity profiles are also shown in Figure 6(a). The pre-disruption current profile is obtained using the motional Stark effect measurements (MSE) as constraints for EFIT.

Since the pre- and post-disruption currents reach different values (full current is $I_{p}=1185 \mathrm{kA}$ vs $180 \mathrm{kA}$ respectively) and have different geometry ( $a=65 \mathrm{~cm}$ vs $43 \mathrm{~cm}$ respectively), their profiles are compared in terms of internal inductance $l_{i}$ and safety factor $q . l_{i}$ is a dimensionless parameter which can be used to characterize the peaking of the current profile: smaller $l_{i}$ corresponds to a flatter current profile. $q$ is useful in estimations of the MHD stability: higher $q$ generally corresponds to a more stable current profile.

As shown in Figure 6(a), all post-disruption current profiles have $l_{i}$ greater by 30$50 \%$ than the pre-disruption current, even the one assuming the flat impurity profile. All $q$ profiles of the RE current are elevated compared to the pre-disruption current, reaching at the edge $q_{a}=12$ vs $q_{a}=3.6$ (Figure $6(\mathrm{~b})$ ).

Comparing internal inductance indicates that the low-current RE beam in the lowresistivity plasma has a more peaked current profile than the pre-disruption plasma. Present experimental observations agree with the modelling predicting the increase of the current profile peaking during disruptions $[56,57]$ and with the experimental results from JET $[64,65]$. However, it is important to note that presently RE current profile cannot not be well-diagnosed via HXR measurements on DIII-D until argon is purged from the RE beam. Recent simulations claim that peaking of the RE current profile can happen after the disruption while REs move inward the beam in the presence of a large fraction of partially ionized high-Z impurities due to the increased convective transport overcoming the diffusive transport [72]. This suggests we cannot determine when the RE current profile peaking happens since we cannot separate the profile peaking during the disruption and the high-Z RE plateau stage.

Analysis of the current profile peaking shows that $l_{i}$ itself does not determine the MHD stability of the RE beam, but has to be considered along with $q$ profile. As found in the experiment, the RE beam has a more stable $q$ profile despite a greater $l_{i}$ than the pre-disruption current, and no MHD instabilities have been detected driven by the beam. Recent experiments with a $1 \mathrm{MA}$ RE beam show that much higher RE current and much lower edge $q_{a}$ are required to drive large-scale MHD instabilities [66].

To deliberately destabilize the RE beam, a large accelerating loop voltage is applied to ramp up the current as presented in the Section 5.

\subsection{Conclusion}

The RE current density profile is measured by taking the moments of the spatially resolved RE energy distribution function. It is found to be more peaked in terms of internal inductance than the pre-disruption current, but has an elevated $q$ profile and much greater $q_{a}$. The peaking of the RE current profile supports the models predicting 
its peaking during the disruption and the high-Z RE plateau stage. No RE-driven MHD instabilities are observed in the experiment likely due to the relatively small RE current and high $q_{a}$.

\section{Internal MHD instability of the RE beam}

\subsection{Motivation}

It is commonly believed that a post-disruption runaway plasma has a more peaked current profile and thus can be prone to the development of current-driven MHD instabilities. As discussed in Section 4, a more peaked RE current profile is indeed measured in DIII-D via diagnosis of the HXR bremsstrahlung radiation. However, the relatively low-current $(180 \mathrm{kA})$ post-disruption $\mathrm{RE}$ beam in the low density background plasma is found to be MHD stable, and is sustained as long as sufficient transformer flux is provided. RE plateaus lasting up to $1.5 \mathrm{~s}$ are routinely observed.

To deliberately destabilize the RE beam large positive and negative loop voltages are applied as shown in Figure 1(right). The applied voltage with a magnitude of $7.5 \mathrm{~V}$ is comparable with the voltage required for a plasma breakdown and greater by factor of 4.5 than the voltage required for a steady-state RE beam in the low density plasma. Two very different RE beam instabilities are observed at positive and negative loop voltages. The analysis of kinetic instabilities driven at the negative voltage is presented separately in Ref. [21]. The instability driven by ramping up current at the large positive voltage is discussed in this Section.

\subsection{Observations of RE beam internal instability}

When a large positive loop voltage is applied to the RE beam and the current ramps up, large-scale oscillations can be seen in the signals of electron cyclotron emission (ECE) and $\mathrm{D}_{\alpha}$ emission as shown in Figure $7(\mathrm{a}, \mathrm{b}, \mathrm{d})$. The ECE signal experiences large drops while the $\mathrm{D}_{\alpha}$ emission shows an opposite behavior with large spikes. This is a clear picture of a RE beam instability. Closer examination reveals that it is also accompanied by the spikes of equatorial line integrated plasma density and spikes of HXR emission from confined REs measured by the GRI as shown in Figure 7(c,e). At the same time, no RE loss is detected (Figure 7(c)). The RE loss to the tokamak wall is measured using a distant HXR scintillating plastic detector [73]. This detector is placed in a few meters from the tokamak and observes volumetric bremsstrahlung emission; interaction between lost REs and the wall material causes fast increase of its signal. Surprisingly no clear change of externally measured magnetic signals is observed. Even the poloidal magnetic signal $B_{p}$ at the high field side (HFS), which is closest to the RE beam limited at the inner wall, is small and incoherent as seen in Figure $7(\mathrm{f})$. Mean frequency of the observed fluctuations is $230 \mathrm{~Hz}$. Fast changes of plasma signals happen within $300 \mu \mathrm{s}$, while longer 'recovery' stages last for 3-6 ms. 


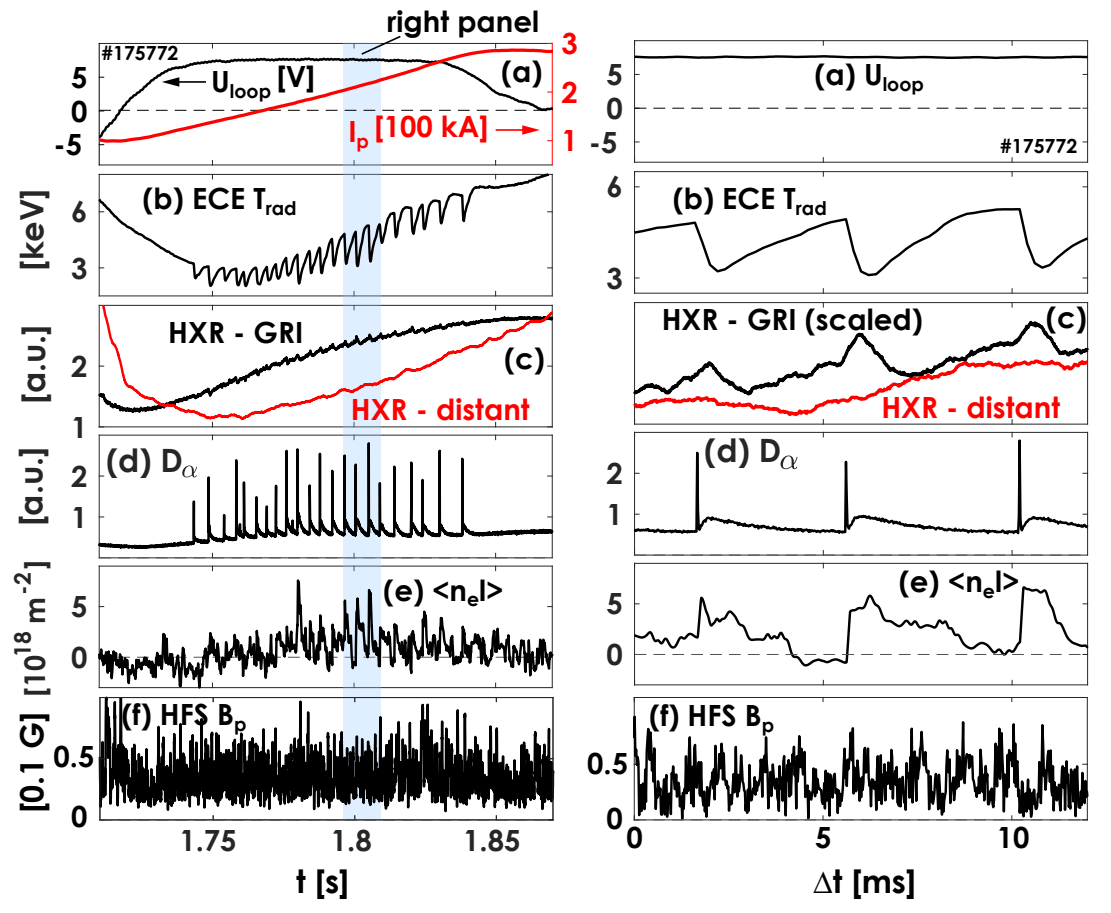

Figure 7: Time traces of (a) loop voltage $\mathrm{U}_{\text {loop }}$ and (a-left) plasma current $\mathrm{I}_{p}$, (b) ECE at 113.5 GHz, (c) HXR signals by GRI and distant detector, (d) $\mathrm{D}_{\alpha}$ signal by filterscopes, (e) equatorial line integrated plasma density $\left\langle n_{e} l\right\rangle$, (f) poloidal magnetic field at HFS.

Even more clear signs of the RE beam instability are observed by a fast visible camera. The camera shows that fluctuations of plasma signals correlate with intense flashes of visible radiation. These flashes are localized in the core of the RE beam and their shape changes from solid circles into bright rings with a radius of $10 \mathrm{~cm}$ as shown in Figure 8(top). It can be also seen that sometimes flashes move along the torus with an approximate speed of $20 \mathrm{~km} / \mathrm{s}$ (Figure 8 (bottom)).

When a positive loop voltage is applied and the $\mathrm{RE}$ current rises, the radius of the $\mathrm{RE}$ beam increases and its core moves with respect to the chords of the $\mathrm{CO}_{2^{-}}$ interferometer. As a result, it is found that the bursts of the plasma density are localized. Moreover, they are co-localized with the radiation flashes as shown in Figure 9(a,b).

The flashes observed by the visible camera are considered to have a non-synchrotron nature. At first, they have a broadband spectrum: seen by the visible camera (with a filter at $790 \mathrm{~nm}$ ), by the IR camera (sensitive to $3-5 \mu \mathrm{m}$ ), and by different spectral channels of filterscopes (photodiodes with filters selecting $\mathrm{D}_{\alpha}, \mathrm{D}_{\beta}$ CI, CII, CIII, HeII spectral lines $[74,75])$. Secondly, these flashes appear to be isotropic since they are seen by the visible camera that looks tangentially in the RE beam forward direction, by the IR camera that looks tangentially in the RE beam reverse direction, and by the filterscopes that have a radial fan of sight lines. 

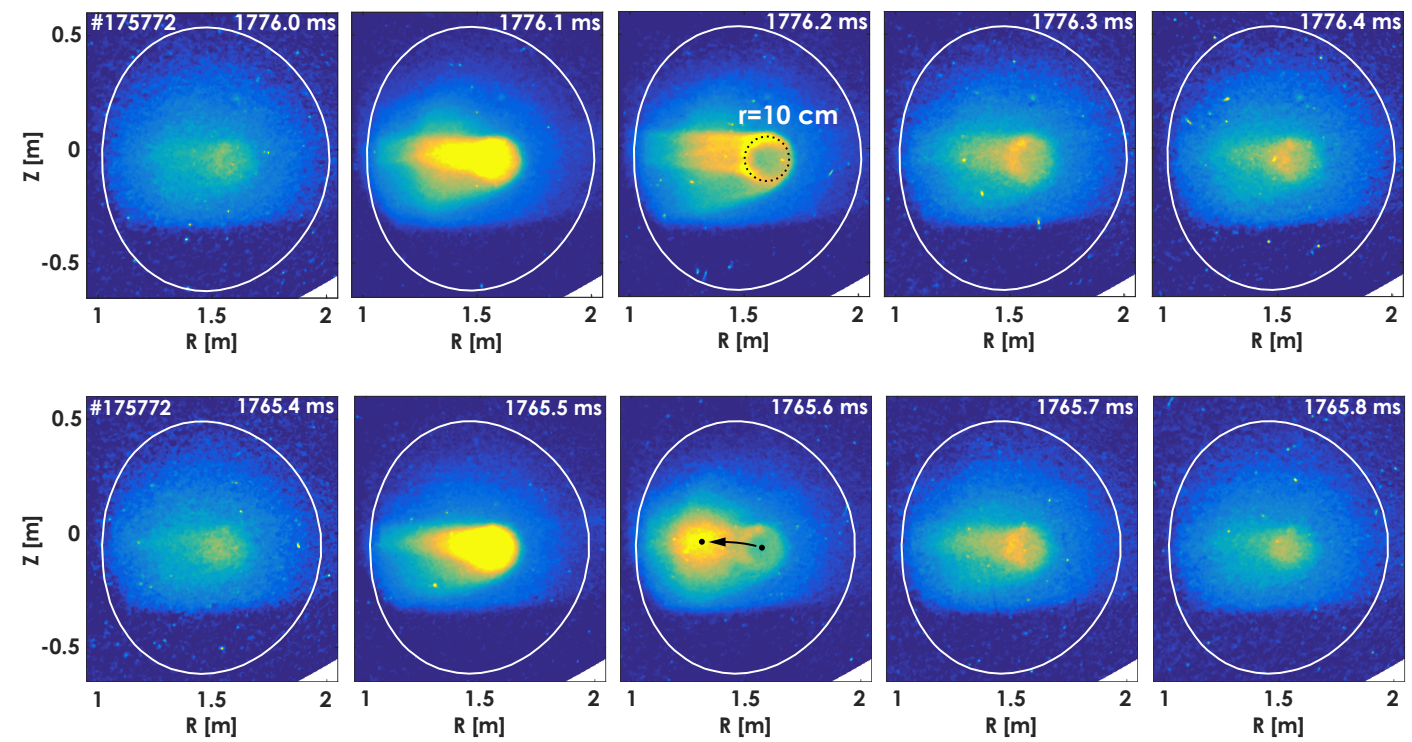

Figure 8: The RE beam instability imaged by the visible fast camera. (top) Formation of the ring-like structure, (bottom) toroidal movement of the radiation flash.
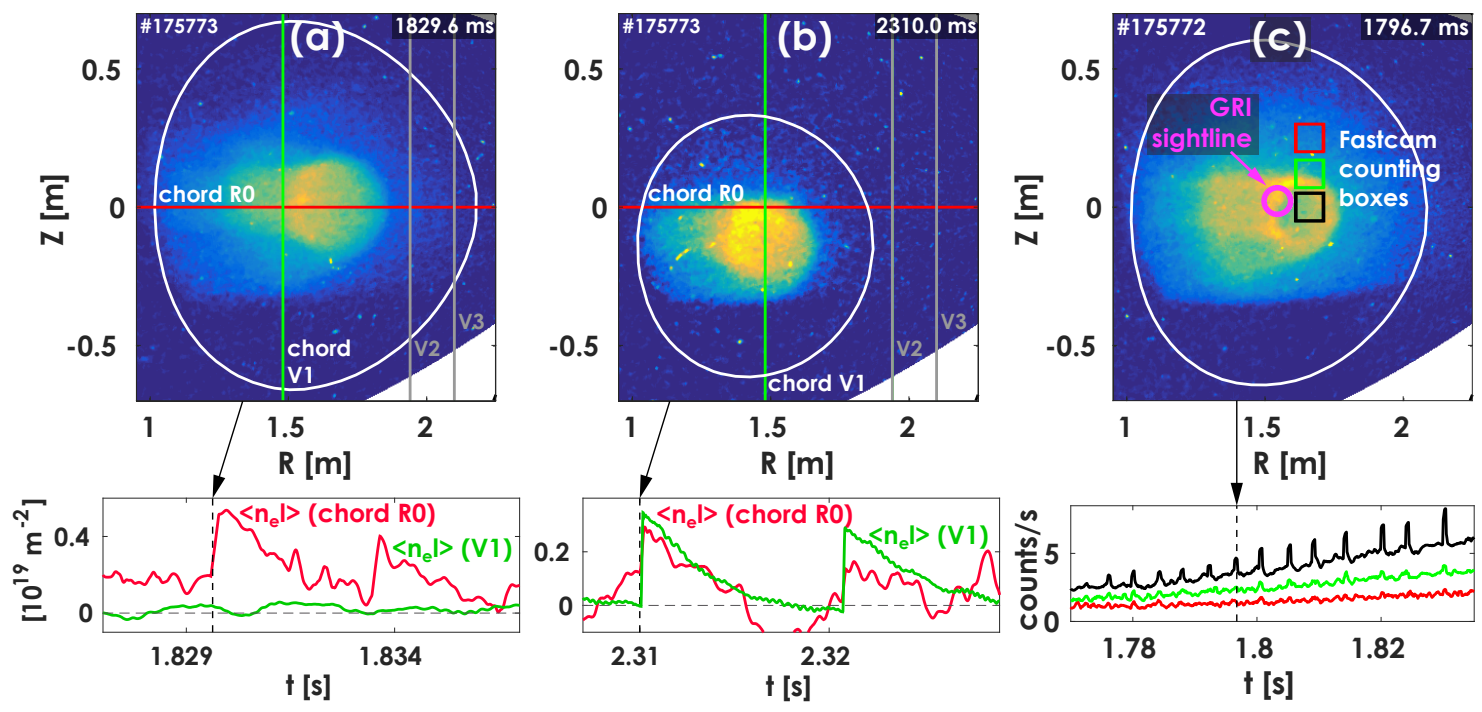

Figure 9: Radiation flashes imaged by the visible camera and $(a, b)$ time traces of line integrated plasma density, (c) time traces of the signal by the visible camera inside the counting boxes.

\subsection{Candidate instability}

We posit that the observed fluctuations are not caused by RE-driven kinetic instabilities. For example, Parail-Pogutse instability [26,76] (also known as 'fan' and anomalous Doppler resonance instability) is often associated with fluctuations of plasma signals when a suprathermal electron population is present in plasma and it is usually accompanied by fast pitch-angle scattering of energetic electrons [36,77-79]. Such an instability would cause spikes of the ECE signal, bursts of the synchrotron radiation, 
drops of the beam-forwarded HXR bremsstrahlung radiation from confined REs and possible spikes of the HXR RE loss signal. None of these is observed in the present experiment. In contrast to Parail-Pogutse instability the measured fluctuations of plasma signals show the opposite dynamics like a fall of ECE and a rise of beamforwarded bremsstrahlung signals.

Excitation of whistler and Alfvénic waves by REs, recently observed in the series of experiments on DIII-D [21,80-82], is usually diagnosed by clear magnetic fluctuations in the $\mathrm{MHz}$ range. Despite the high-frequency (digitized at $200 \mathrm{MHz}$ ) magnetic diagnostic is enabled in the present experiment, such magnetic fluctuations are not found. Spikes of the GRI signal, Figure 8(c), are seen in RE spectra as the overall increase of the RE number, therefore we do not interpret them as evidences of modification of the $\mathrm{RE}$ distribution function due to resonant $\mathrm{RE}$-wave interactions similar to observations reported in Ref. [21].

Though there is still a chance that these fluctuations can be caused by kinetic instabilities present in the space not accessible by DIII-D diagnostics, we propose a core-localized 1/1 MHD instability as a candidate instability which is most reasonably supported by the available measurements. Its driving mechanism can be as follows.

A large applied loop voltage accelerates the RE beam and can lead to formation of a peaked current profile. According to simulations of RE beam stability [60,61], a peaked current profile can excite internal kink modes which causes the relaxation of the current profile. Such sawtooth-like redistribution of the RE current can explain the observed drops of ECE signals, bursts of the broadband radiation and increase of the local plasma density. The latter is possibly due to the Ohmic drive replacing the decreasing RE current in the core. Redistribution of the RE current can also explain the lack of detected RE loss but observed increase of the GRI signal as shown in Figures 7(c) and $9(\mathrm{c})$. Finally, excitation of the $1 / 1$ mode in the RE beam core can explain poorly detectable external magnetic signals.

We would like to note, that the method to reconstruct the RE current density (and safety factor) profile via the vertical scan of the RE beam, presented in Section 4, has relatively low time resolution (set by the vertical scan rate), thus it is not applicable on the timescales of the present experiment. It requires about $200-300 \mathrm{~ms}$ to provide a single profile, while the RE current changes at a rate about $150 \mathrm{kA} / 100 \mathrm{~ms}$ and the instability is observed every $3-6 \mathrm{~ms}$. Despite no direct measurements of the current profile are available, signals by the visible camera can be considered as a proxy of profile peaking: their intensity increases in the core much faster than at the edge as shown in Figure 9(c).

\subsection{MARS-F ideal modelling}

To overcome the lack of magnetic signatures measured in the experiment, the excitation of proposed 1/1 mode is modelled using the MARS-F code [83]. To do this, the RE current profile measured by the GRI via vertical scan in the discharge 175768 is used to 

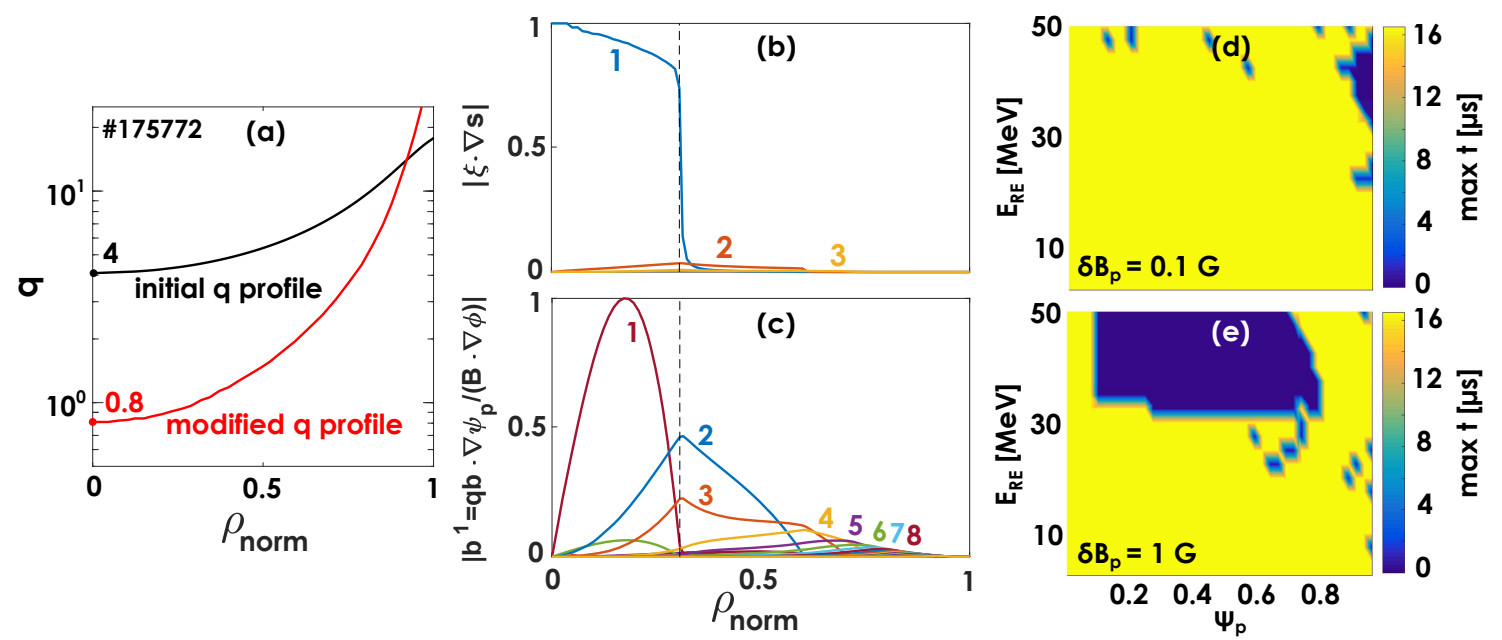

Figure 10: (a) $q$ profile of the RE beam calculated by EFIT with current profile constraints and modified peaked $q$ profile. MARS-F modelling of internal kink modes: (b) radial displacement and (c) poloidal magnetic field; calculated up to 32 modes, only visible modes are shown. MARS-F modelling of prompt RE loss under applied perturbations of poloidal magnetic field: (d) $\delta B_{p}=0.1 \mathrm{G}$ and (e) $\delta B_{p}=1 \mathrm{G}$.

constrain the current profile in EFIT reconstructions of the analyzed discharge 175772. As a result, EFIT successfully converges and the current profile is obtained before the current is ramped up. This initial current profile is modified to achieve $q_{0}<1$ (chosen $q_{0}=0.8$ ) while the total current $\mathrm{I}_{\mathrm{p}}$ is kept constant as shown in Figure 10(a). The calculated eigenfunctions of $n=1$ internal kink clearly show the excitation of a large 1/1 mode as presented in Figure 10(b,c). Despite up to 32 modes are calculated, no magnetic signal at the edge is found in the simulations, supporting the observed lack of externally measured magnetic modes.

A prompt (on a $\mu$ s time scale) RE loss domain is computed assuming $n=1$ internal kink mode eigenfunction using a drift orbit model for relativistic test electrons recently incorporated into the MARS-F code [84]. Almost no RE loss is found when perturbations of the poloidal magnetic field with a magnitude $\delta B_{\mathrm{p}}=0.1 \mathrm{G}$ are applied at the HFS inside the DIII-D vessel as shown in Figure 10(d). When even much larger perturbations are applied with $\delta B_{\mathrm{p}}=1 \mathrm{G}$, RE loss is significant only for REs with energy above $30 \mathrm{MeV}$ (Figure 10(e)), which is in contrast to an external kink, usually increasing loss of low-energy REs. Simulations show that the large 1/1 internal kink mode creates a stellarator-like magnetic field structure which traps low-energy REs in the core. Trajectories of high-energy REs are less affected by this trapping effect, but sensitive to weaker modes in the outer perturbation region, like $2 / 1$ and $3 / 1$ modes, scattering high-energy REs from the plasma. In summary, these results support the observations of no RE loss driven by the internal kink in the experiment. 


\subsection{Conclusion}

Large accelerating loop voltage applied to the RE beam presumably modifies the RE current profile which leads to the excitation of core-localized MHD instabilities. MARS$\mathrm{F}$ modelling of the peaked current profile suggests formation of the internal 1/1 kink mode. These results support previous theoretical finding predicting the possibility of internal kink modes driven by a peaked RE current profile. We have to note that this is achieved under deliberately modified experimental conditions usually not observed during the RE plateau stage, though important conclusions can be made. The RE beam internal instability at a large edge safety factor causes no RE loss both in the experiment and in the modelling and thus provides no positive outcome of the internal kink mode for RE mitigation. On the contrary, MHD unstable RE current profiles are likely best avoided since large-scale MHD instabilities can lead to minor and eventually major disruption of the RE beam as discussed in Ref. [66].

\section{Summary}

The energy distribution function, current density profile, and internal MHD instability of the post-disruption RE beam in the low-density plasma achieved after the secondary deuterium injection have been investigated in DIII-D.

The RE energy distribution function has been measured during the RE plateau stage by inverting the hard X-ray bremsstrahlung spectra. It has the maximum energy up to $20 \mathrm{MeV}$ and the non-monotonic feature (bump) at 5-6 MeV observed only in the core supporting the possibility of kinetic instabilities. Conservation of the distribution function has been found over the whole observation period of $450 \mathrm{~ms}$, which can be explained by the balance between the small accelerating electric field and the collisional damping on bound electrons introduced by the deuterium injection. These observations are consistent with 0D-2V Fokker-Plank modelling of the time-dependent energy distribution function.

The spatially resolved RE energy distribution function has been obtained for the first time in the post-disruption conditions via the vertical scan of the RE beam. The reconstructed RE current profile has been found to be more peaked than the pre-disruption plasma current, with higher internal inductance, suggesting preferential formation of REs in the core plasma or potentially a radially inward motion of REs. Despite the peaked current profile, the relatively low-current (180 kA) RE beam has been found stable, likely due to its elevated safety factor profile, and it sustains as long as there is enough transformer flux provided. This scenario, developed to access the RE current profile measurements, can be used in future to investigate the formation and evolution of the RE current profile.

The RE beam has been found to be MHD unstable during the current ramp up under applied large accelerating loop voltage. An internal instability presumably driven by the peaked current profile and leading to sawtooth-like relaxation events has been 
observed. The MARS-F modelling suggests excitation of the 1/1 internal kink mode with a vanishing external magnetic component, which could explain the experimental results. Most importantly, this instability has been found to drive no significant RE loss, consistent with MARS-F modelling of perturbed RE drift orbits. This indicates that effect of the mode can be neglected in simulations of the RE dissipation at large edge safety factor.

Though these results have been obtained under the unusual conditions of the postdisruption RE plateau such as the low density background plasma and significantly modified loop voltage they are useful for understanding the RE equilibrium and stability, and provide new input to verify models predicting the physics of REs in ITER.

\section{Acknowledgments}

Authors are thankful for diagnostic support to I. Bykov, C. Lasnier, T. Strait, and B. Wilcox. Discussion with C. Liu and D. Brennan is appreciated. This material is based upon work supported by the U.S. Department of Energy, Office of Science, Office of Fusion Energy Sciences, using the DIII-D National Fusion Facility, a DOE Office of Science user facility, under U.S. DOE Frontier Science Program and Award DE-FC0204ER54698.

\section{Disclamer}

This report was prepared as an account of work sponsored by an agency of the United States Government. Neither the United States Government nor any agency thereof, nor any of their employees, makes any warranty, express or implied, or assumes any legal liability or responsibility for the accuracy, completeness, or usefulness of any information, apparatus, product, or process disclosed, or represents that its use would not infringe privately owned rights. Reference herein to any specific commercial product, process, or service by trade name, trademark, manufacturer, or otherwise, does not necessarily constitute or imply its endorsement, recommendation, or favoring by the United States Government or any agency thereof. The views and opinions of authors expressed herein do not necessarily state or reflect those of the United States Government or any agency thereof.

\section{Appendix A. Electron cyclotron emission during RE plateau stage}

ECE produced by REs consists of many close-spaced and overlapping harmonics, as a result it cannot be straightforwardly interpreted and used similar to the ECE from thermal electrons. Nevertheless, it can still provide insights into the physics of REs via comparison with models. For example, the shape and the evolution of ECE spectrum

produced by REs in low-density discharges on DIII-D can be explained by simulations if 
kinetic instabilities are taken into account [85]. In this Appendix we present observations of the bifurcated ECE spectrum measured during the RE plateau stage.

ECE is detected in DIII-D by a heterodyne reflectometer in the range 83.5$129.5 \mathrm{GHz}$ [43]. For typical plasma parameters, this provides ECE measurements at the dominant second harmonic $\mathrm{X}$-mode with unambiguous frequency dependence on the major radius (Figure A1(a)). ECE measurements during the RE plateau stage are usually non-accessible, since impurity injection used to trigger the disruption significantly increases the plasma density and leads to the ECE cut-off. The novel scenario described in Section 2 provides very low density plasma $\left(n_{e}<5 \cdot 10^{18} \mathrm{~m}^{-3}\right.$, $f_{\mathrm{pe}}<20 \mathrm{GHz}$ ), which allows ECE leaving the plasma as shown in Figure 1(c,d).

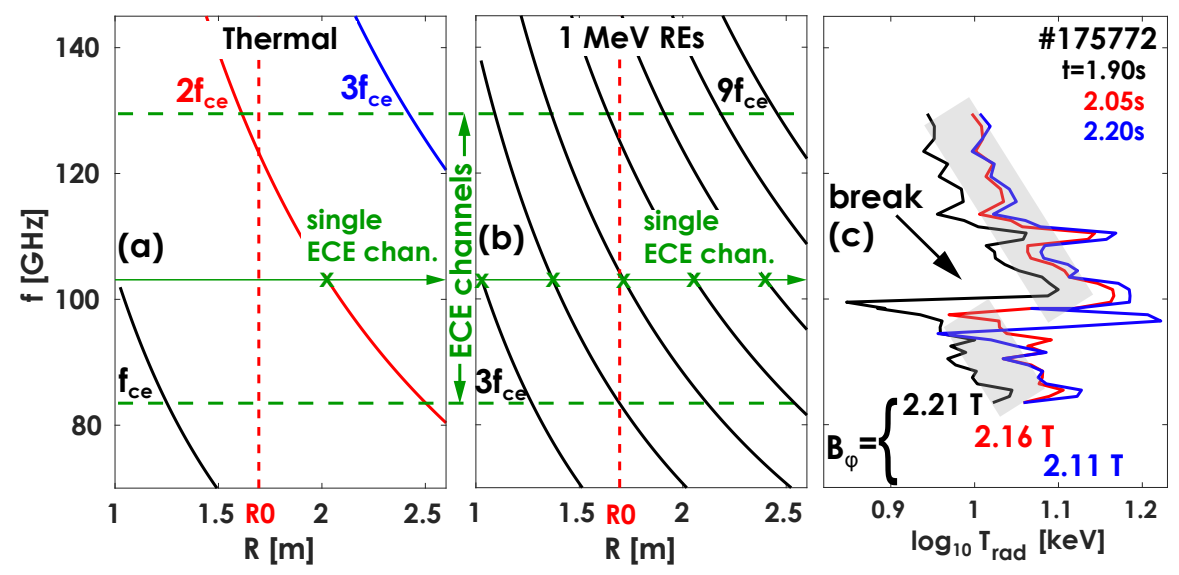

Figure A1: (a) ECE produced by thermal electrons and the ECE range diagnosed in DIII-D, (b) example of ECE produced by $1 \mathrm{MeV}$ REs, (c) bifurcated ECE measured during the RE plateau stage at different $\mathrm{B}_{\phi}$.

ECE produced by REs is difficult to interpret since it consists of numerous overlapping harmonics due to relativistic mass increase $f_{c e} \propto\left(\gamma m_{e}\right)^{-1}$ and depends on the pitch-angle of REs. An example of emission from mono-energetic $1 \mathrm{MeV}$ REs is shown in Figure A1(b). The RE ECE spectrum misses the unambiguous $f_{\text {ce }}(R)$ dependence and expected to be nearly flat over observed frequencies. Contradicting to these expectations, the intensity of the ECE spectrum measured during the RE plateau stage is not flat but increases while the frequency decreases and also has a clear break point at $\approx 100 \mathrm{GHz}$ (Figure A1(c)). The break point location depends on the toroidal magnetic field $B_{\phi}$ and shifts to lower frequencies when $B_{\phi}$ decreases (Figure A1(c)). This feature of the RE ECE spectrum is presently unclear. Hypothetically, it can be caused by some resonant damping at the first thermal ECE harmonic, yet a detailed investigation of its specific mechanism is necessary.

The ECE spectrum shown in Figure A1(c) can be presented by high- and lowfrequency parts separated by the break point. Fluctuations of the ECE signal observed during the internal kink (Section 5) and frequency chirping (Ref. [21]) instabilities, both driven by REs, have very different spectral patterns. The internal kink instability correlates with a crash of the ECE signal over all frequencies, presumably due to 

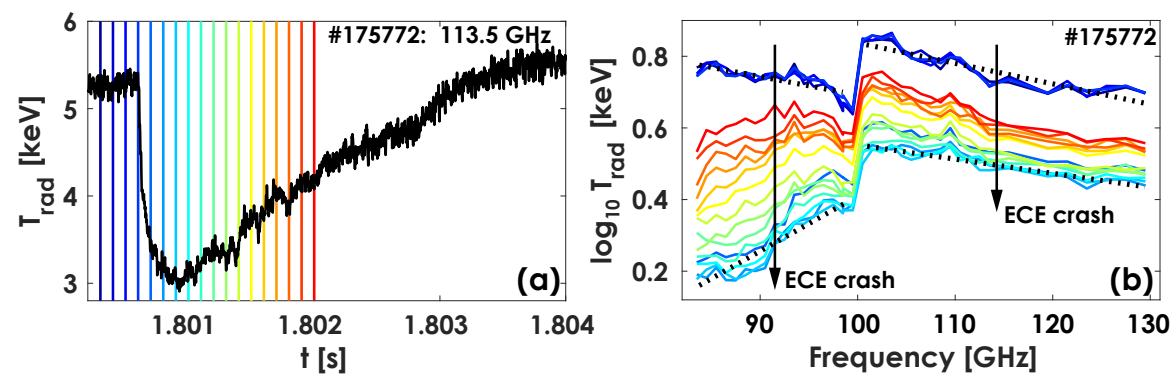

Figure A2: ECE crash during the internal kink instability (Section 5).
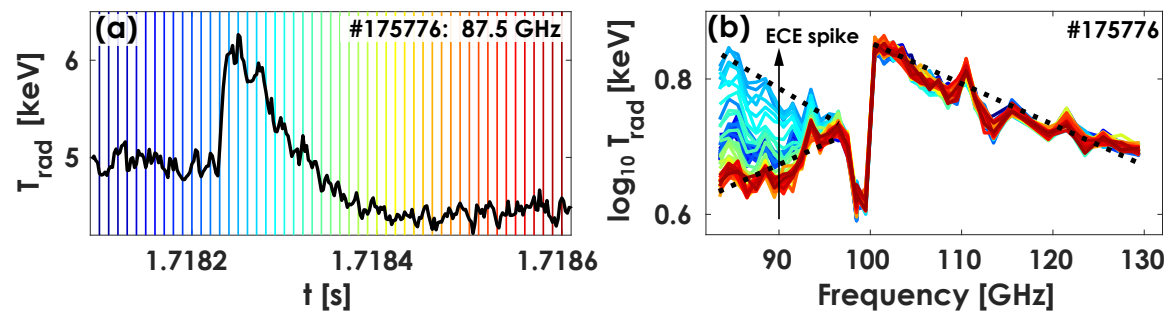

Figure A3: ECE spike during the frequency chirping instability (Ref. [21]).

sawtooth-like relaxation of the current profile (Figure A2(a)). However, the highfrequency part of the spectrum reduces nearly by the same value, while the low-frequency part experiences a much larger decrease (Figure A2(b)). The density bursts observed during the RE current profile relaxation (Figure 9(a,b)) cannot cause noticeable ECE cut-off and explain this picture, since they increase the plasma frequency only up to 50 $\mathrm{GHz}$. The frequency chirping instabilities correlate with the spikes of ECE emission, likely due to the fast pitch-angle scattering of REs. However, this sudden increase of the ECE signal is observed only at low frequencies (Figure A3(b)).

These new experimental observations can provide a valuable input for models predicting ECE from REs.

\section{Appendix B. HXR measurements during the RE plateau}

\section{Pile-up effect}

The $\mathrm{MHz}$ counting rate is the practical operating limit of the LYSO detector. At first, the detector underestimates the energy of incident gammas when the gamma flux approaches to the $\mathrm{MHz}$ counting rate [41]. Secondly, it underestimates the energy of incident gammas when they exceed a few MeV. Both these effects can be compensated using calibrating curves similar to that presented in Ref. [41]. This requires measurements of the flux and energy of gammas not affected by other factors such as, for example, pile-up because it can worsen the energy resolution and discrimination of single gammas during the pulse height analysis (PHA). Since the PHA is able to discriminate single pulses if their peaks are separated by more than $35 \mathrm{~ns}$, the fraction of pulses missed due to the pile-up [86] is about $15 \%$ owing the maximum counting 

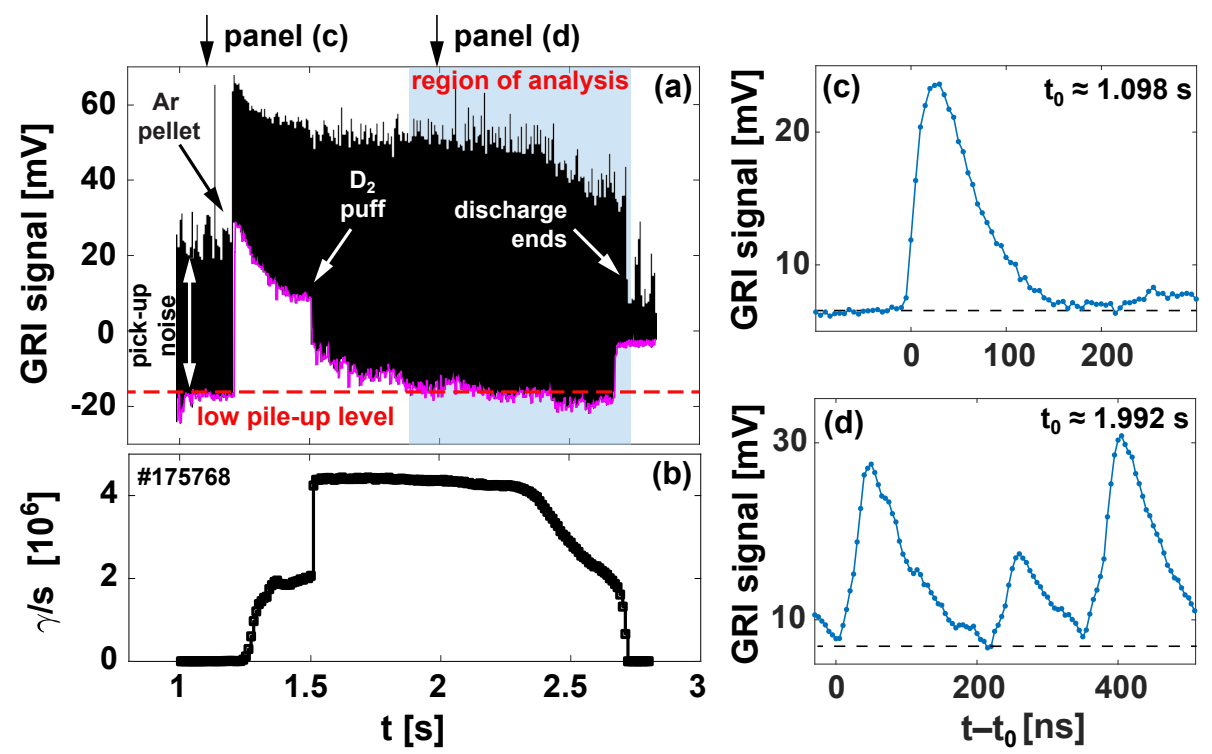

Figure B1: (a) Raw signal waveform measured by the GRI LYSO detector. (b) Gamma flux resolved via pulse height analysis. (c) A single gamma pulse measured before the disruption likely produced by activated materials of DIII-D. (d) Typical picture of gamma pulses measured during the RE plateau stage at the low pile-up level.

rate of $4.3 \mathrm{MHz}$ measured in the experiment. Though, in case of high peak pile-up and poor discrimination of single gamma pulses, the gamma flux resolved via PHA can become completely unreliable and seem to be below this value, as before the $\mathrm{D}_{2}$ puff in Figure B1(b). Thus, as an additional metric of a low pile-up we use the moving minimum of the raw LYSO signal as shown in magenta in Figure B1(a). The raw signal before the RE beam stage mostly presents strong $\mathrm{kHz}$ magnetic pickup noise and has zero pile-up. The raw signal during the RE beam stage consists of the same noise and numerous gamma pulses. When the pulses strongly pile up, as after the argon pellet injection, the moving minimum shifts up. When the moving minimum reduces to its pre-disruption value, as after the argon purge from the plasma, we assume that the pile-up level becomes small enough to do not lead to completely unreliable PHA results. A zoom in to the raw signal during that period, Figure B1(d), supports this assumption. Considering the requirement on the low pile-up level for the reliable operation of the LYSO detector, its measurements are analyzed and assumed to be valid in $400 \mathrm{~ms}$ after the argon purge, during the last $800 \mathrm{~ms}$ of the RE plateau, Figure B1(a).

\section{Collimation effect}

The HXR flux, exceeding the $\mathrm{MHz}$ counting rate during the RE plateau stage, is still strong enough to be detected above the noise level when the RE beam is out of the collimated sight line (after $t=2.55 \mathrm{~s}$, see Figures $4(\mathrm{e}, \mathrm{c}, \mathrm{d})$ and $\mathrm{B} 1(\mathrm{~b})$ ). As discussed in detail in Section 4, the insufficient collimation has to be taken into account for reconstruction of the $\mathrm{RE}$ current density profile. However, we assume that this is not the factor explaining the observed non-monotonic feature. The bump still persists if the 

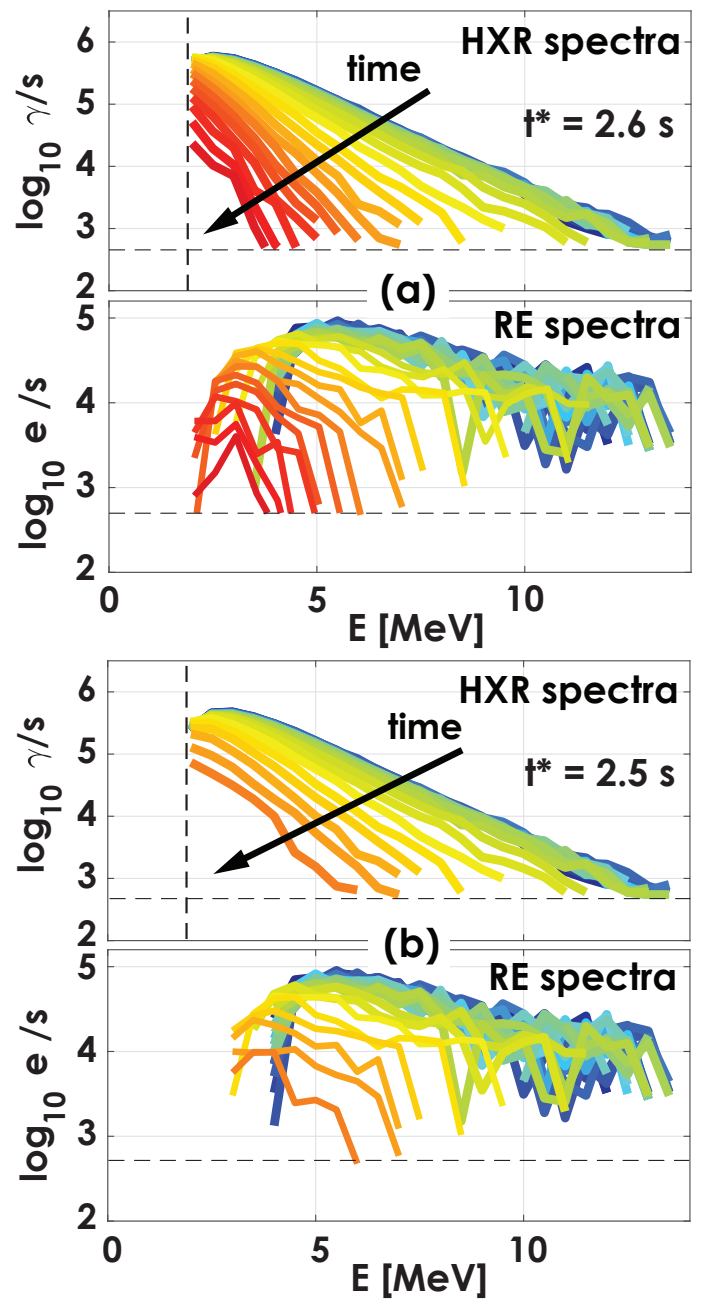

Figure B2: (a,b) HXR and RE spectra when the HXR spectrum (obtained at $t^{*}=2.6$ $\mathrm{s}$ and $t^{*}=2.5 \mathrm{~s}$ accordingly) is subtracted. Time slices are color-coded same as in Figure 2.

HXR spectrum measured at $t=2.6 \mathrm{~s}$ (when the beam is out of sight) is subtracted from all previous HXR spectra and then an inversion to RE spectra takes place, as shown in Figure B2(a). Moreover, the bump is observed even if the HXR spectrum measured at $t=2.5 \mathrm{~s}$ (when the detector images the edge of the beam, $r / a \approx 0.9$ ) is subtracted, Figure B2(b).

\section{Instrumental effect}

An instrumental effect is not considered as a factor causing the observation of nonmonotonic features in RE plateau and QRE experiments what we justify as follows. At first, detectors with different crystals (BGO vs LYSO) and different read-out electronics were used in these experiments. Secondly, the bump is not always observed in the RE beam experiment: it shifts to lower energy and finally disappears when the part of the RE beam imaged by the detector changes from the core to the edge, Figure 2(c) (due to the mentioned earlier vertical movement of the beam which is shown in Figure 4(e) 
and discussed in detail in Section 4). Thirdly, the bump is also not always observed in the QRE experiment: the bump appears in the course of time when the RE population becomes more energetic, as shown in Figure 2(f) in Ref. [24].

\section{References}

[1] Lehnen M, Aleynikova K, Aleynikov P B, Campbell D J, Drewelow P, Eidietis N W, Gasparyan Y, Granetz R S, Gribov Y, Hartmann N, Hollmann E M, Izzo V A, Jachmich S, Kim S H, Kočan M, Koslowski H R, Kovalenko D, Kruezi U, Loarte A, Maruyama S, Matthews G F, Parks P B, Pautasso G, Pitts R A, Reux C, Riccardo V, Roccella R, Snipes J A, Thornton A J and De Vries P C 2015 Journal of Nuclear Materials 463 39-48 ISSN 00223115 URL http://www.sciencedirect.com/science/article/pii/S0022311514007594

[2] Hollmann E M, Aleynikov P B, Fülöp T, Humphreys D A, Izzo V A, Lehnen M, Lukash V E, Papp G, Pautasso G, Saint-Laurent F and Snipes J A 2015 Physics of Plasmas 22021802 ISSN 1070-664X URL http://aip.scitation.org/doi/10.1063/1.4901251

[3] Lehnen M, Campbell D J, Hu D, Kruezi U and Luce T C R\\&D for reliable disruption mitigation in ITER Preprint: 2018 IAEA Fusion Energy Conference, Ahmedabad, India [EX/Pr-12] URL https://conferences.iaea.org/indico/event/151/papers/6095/files/ 4605-IAEA \{_\} 2018\{_\}ITER \{-\}DMS \{_\}RD \{_\} paper\{_\}v1.4.pdf

[4] Paz-Soldan C, Eidietis N W N, Hollmann E M E, Aleynikov P, Carbajal L, Heidbrink W W, Hoppe M, Liu C, Lvovskiy A, Shiraki D, Spong D A, Brennan D P D, Cooper C C M, DelCastillo-Negrete D, Du X, Embreus O, Fulop T, Herfindal J, Moyer R A, Parks P B and Thome K K E 2019 Nuclear Fusion 59066025 ISSN 0029-5515 URL http://iopscience.iop.org/article/ 10.1088/1741-4326/ab1769

[5] Breizman B N, Aleynikov P, Hollmann E M and Lehnen M 2019 Nuclear Fusion 59083001 ISSN 0029-5515 URL http://iopscience.iop.org/article/10.1088/1741-4326/ab1822

[6] Eidietis N W, Commaux N, Hollmann E M, Humphreys D A, Jernigan T C, Moyer R A, Strait E J, VanZeeland M A, Wesley J C and Yu J H 2012 Physics of Plasmas 19056109 ISSN 1070664X URL http://scitation.aip.org/content/aip/journal/pop/19/5/10.1063/1.3695000

[7] Esposito B, Boncagni L, Buratti P, Carnevale D, Causa F, Gospodarczyk M, Martin-Solis J, Popovic Z, Agostini M, Apruzzese G, Bin W, Cianfarani C, De Angelis R, Granucci G, Grosso A, Maddaluno G, Marocco D, Piergotti V, Pensa A, Podda S, Pucella G, Ramogida G, Rocchi G, Riva M, Sibio A, Sozzi C, Tilia B, Tudisco O, Valisa M and Team F 2017 Plasma Physics and Controlled Fusion 59014044 ISSN 0741-3335 URL http://stacks.iop.org/0741-3335/59/i= $1 / \mathrm{a}=014044$

[8] Saint-Laurent F, Martin G, Alarcon T, Le Luyer A, Parks P B, Pastor P, Putvinski S, Reux C, Bucalossi J, Bremond S and Moreau P 2013 Fusion Science and Technology 64 711-718 ISSN 1536-1055 URL https://www.tandfonline.com/doi/full/10.13182/FST13-A24090

[9] Carnevale D, Ariola M, Artaserse G, Bagnato F, Bin W, Boncagni L, Bolzonella T, Bombarda F, Buratti P, Calacci L, Causa F, Coda S, Cordella F, Decker J, Tommasi G D, Duval B, Esposito B, Ferrò G, Ficker O, Gabellieri L, Gabrielli A, Galeani S, Galperti C, Garavaglia S, Havranek A, Gobbin M, Gospodarczyk M, Granucci G, Joffrin E, Lennholm M, Lier A, Macusova E, Martinelli F, Martìn-Solìs J R, Mlynar J, Panaccione L, Papp G, Passeri M, Pautasso G, Popovic Ž, Possieri C, Pucella G, Sheikh U A, Ramogida G, Reux C, Rimini F, Romano A, Sassano M, Tilia B, Tudisco O, Valcarcel D, Team t F, Team t E M and Contributors J 2019 Plasma Physics and Controlled Fusion 61014036 ISSN 0741-3335 URL http://stacks.iop.org/0741-3335/61/i=1/a=014036

[10] Hollmann E M, Austin M E, Boedo J A, Brooks N H, Commaux N, Eidietis N W, Humphreys D A, Izzo V A, James A N, Jernigan T C, Loarte A, Martin-Solis J, Moyer R A, Muñoz-Burgos J M, Parks P B, Rudakov D L, Strait E J, Tsui C, Van Zeeland M A, Wesley J C and Yu J H 
RE beam in DIII-D: energy, current profile and internal instability

2013 Nuclear Fusion 53083004 ISSN 0029-5515 URL http://stacks.iop.org/0029-5515/53/i=8/ $\mathrm{a}=083004$

[11] Lvovskiy A, Koslowski H R and Zeng L 2015 Journal of Plasma Physics 81475810507 ISSN 0022-3778 URL http://www.journals.cambridge.org/abstract\{_\}S0022377815001051

[12] Reux C, Plyusnin V, Alper B, Alves D, Bazylev B, Belonohy E, Boboc A, Brezinsek S, Coffey I, Decker J, Drewelow P, Devaux S, de Vries P, Fil A, Gerasimov S, Giacomelli L, Jachmich S, Khilkevitch E, Kiptily V, Koslowski R, Kruezi U, Lehnen M, Lupelli I, Lomas P, Manzanares A, Martin De Aguilera A, Matthews G, Mlynář J, Nardon E, Nilsson E, Perez von Thun C, Riccardo V, Saint-Laurent F, Shevelev A, Sips G, Sozzi C and Contributors J 2015 Nuclear Fusion 55093013 ISSN 0029-5515 URL http://stacks.iop.org/0029-5515/55/i=9/a=093013

[13] Pautasso G, Bernert M, Dibon M, Duval B, Dux R, Fable E, Fuchs J C, Conway G D, Giannone L, Gude A, Herrmann A, Hoelzl M, McCarthy P J, Mlynek A, Maraschek M, Nardon E, Papp G, Potzel S, Rapson C, Sieglin B, Suttrop W, Treutterer W, Team T A U and Team T E M 2017 Plasma Physics and Controlled Fusion 59014046 ISSN 0741-3335 URL http://stacks.iop.org/0741-3335/59/i=1/a =014046

[14] Zeng L, Chen Z, Dong Y, Koslowski H, Liang Y, Zhang Y, Zhuang H, Huang D and Gao X 2017 Nuclear Fusion 57046001 ISSN 0029-5515 URL http://stacks.iop.org/0029-5515/57/i=4/a= 046001

[15] Shiraki D, Commaux N, Baylor L, Cooper C, Eidietis N, Hollmann E, Paz-Soldan C, Combs S and Meitner S 2018 Nuclear Fusion 58056006 ISSN 0029-5515 URL http://stacks.iop.org/ $0029-5515 / 58 / \mathrm{i}=5 / \mathrm{a}=056006$

[16] Hollmann E, Eidietis N, Herfindal J, Parks P, Pigarov A, Shiraki D, Austin M, Bardoczi L, Baylor L, Bykov I, Carlstrom T, Kaplan D, Lasnier C, Lvovskiy A, Moser A, Moyer R, Paz-Soldan C, Rudakov D, Samuell C, Shafer M, Van Zeeland M, Welander A and Wilcox R 2019 Nuclear Fusion 59106014 ISSN 0029-5515 URL http://iopscience.iop.org/article/10.1088/1741-4326/ ab32b2

[17] Commaux N, Baylor L, Combs S, Eidietis N, Evans T, Foust C, Hollmann E, Humphreys D, Izzo V, James A, Jernigan T, Meitner S, Parks P, Wesley J and Yu J 2011 Nuclear Fusion 51103001 ISSN 0029-5515 URL http://stacks.iop.org/0029-5515/51/i=10/a=103001

[18] Koslowski H R, Zeng L, Lehnen M, Lvovskiy A and Wongrach K 2014 Influence of massive gas injection and resonant magnetic perturbations on the generation of runaway electrons during disruptions in TEXTOR Preprint: 2014 EPS Conference on Plasma Physics, Berlin, Germany [P5.028] URL http://ocs.ciemat.es/EPS2014PAP/pdf/P5.028.pdf

[19] Gobbin M, Li L, Liu Y Q, Marrelli L, Nocente M, Papp G, Pautasso G, Piovesan P, Valisa M, Carnevale D, Esposito B, Giacomelli L, Gospodarczyk M, McCarthy P J, Martin P, Suttrop W, Tardocchi M, Teschke M, Team t A U and Team t E M 2018 Plasma Physics and Controlled Fusion 60014036 ISSN 0741-3335 URL http://stacks.iop.org/0741-3335/60/i=1/a=014036

[20] Lin Z F, Chen Z Y, Huang D W, Huang J, Tong R, Wei Y N, Yan W, Li D, Hu Q M, Huang Y, Yang H Y, Li Y, Zhang X Q, Rao B, Yang Z J, Gao L, Ding Y H, Wang Z J, Zhang M, Liang Y, Pan Y, Jiang Z H and Team J T 2019 Plasma Physics and Controlled Fusion 61024005 ISSN 0741-3335 URL http://stacks.iop.org/0741-3335/61/i=2/a=024005

[21] Lvovskiy A, Heidbrink W W, Paz-Soldan C, Spong D A, Dal Molin A, Eidietis N W, Nocente M, Shiraki D and Thome K E 2019 Nuclear Fusion 59124004 ISSN 0029-5515 URL http: //iopscience.iop.org/article/10.1088/1741-4326/ab4405

[22] Evans T E, Taylor P L and Whyte D G 1998 Preprint: 1998 IAEA Fusion Energy Conference, Yokohama, Japan [EXP3/07] URL https://inis.iaea.org/collection/NCLCollectionStore/\{_\} Public/31/011/31011383.pdf?r=1 $\{\&\} \mathrm{r}=1$

[23] Hollmann E M, Commaux N, Eidietis N W, Evans T E, Humphreys D A, James A N, Jernigan T C, Parks P B, Strait E J, Wesley J C, Yu J H, Austin M E, Baylor L R, Brooks N H, Izzo V a, Jackson G L, van Zeeland M A and Wu W 2010 Physics of Plasmas 17056117 ISSN 1070664X URL http://scitation.aip.org/content/aip/journal/pop/17/5/10.1063/1.3309426 
[24] Paz-Soldan C, Cooper C M, Aleynikov P, Pace D C, Eidietis N W, Brennan D P, Granetz R S, Hollmann E M, Liu C, Lvovskiy A, Moyer R A and Shiraki D 2017 Physical Review Letters 118 255002 ISSN 0031-9007 URL http://link.aps.org/doi/10.1103/PhysRevLett.118.255002

[25] Paz-Soldan C, Cooper C M, Aleynikov P, Eidietis N W, Lvovskiy A, Pace D C, Brennan D P, Hollmann E M, Liu C, Moyer R A and Shiraki D 2018 Physics of Plasmas 25056105 ISSN 1070-664X URL http://aip.scitation.org/doi/10.1063/1.5024223

[26] Parail V and Pogutse O 1978 Nuclear Fusion 18303 ISSN 0029-5515 URL http://iopscience.iop. org/article/10.1088/0029-5515/18/3/001

[27] Swanson D G 2003 Plasma waves 2nd ed (Bristol and Philadelphia: Institute of Physics Publishing) ISBN 9780750309271 URL https://www.crcpress.com/Plasma-Waves/Swanson/p/ book/9780750309271

[28] Hollmann E M, Parks P B, Commaux N, Eidietis N W, Moyer R A, Shiraki D, Austin M E, Lasnier C J, Paz-Soldan C and Rudakov D L 2015 Physics of Plasmas 22056108 ISSN 1070-664X URL http://scitation.aip.org/content/aip/journal/pop/22/5/10.1063/1.4921149

[29] Nocente M, Shevelev A, Giacomelli L, Pautasso G, Tardocchi M, Gin D, Gobbin M, Gorini G, Fernandes A, Herrmann A, Khilkevitch E, Panontin E, Papp G, Pereira R C, Salewski M, Tardini G, Valisa M, Team A U and Team E M 2018 Review of Scientific Instruments 89 10I124 ISSN 0034-6748 URL http://aip.scitation.org/doi/10.1063/1.5036658

[30] Plyusnin V V, Reux C, Kiptily V G and Shevelev A E Parameters of Runaway Electrons in JET Preprint: 2014 IAEA Fusion Energy Conference, Saint Petersburg, Russia [EX/P523] URL http://www-naweb.iaea.org/napc/physics/FEC/FEC2014/fec2014-preprints/486\{-\} EXP523.pdf

[31] Plyusnin V, Reux C, Kiptily V, Pautasso G, Decker J, Papp G, Kallenbach A, Weinzettl V, Mlynar J, Coda S, Riccardo V, Lomas P, Jachmich S, Shevelev A, Alper B, Khilkevitch E, Martin Y, Dux R, Fuchs C, Duval B, Brix M, Tardini G, Maraschek M, Treutterer W, Giannone L, Mlynek A, Ficker O, Martin P, Gerasimov S, Potzel S, Paprok R, McCarthy P J, Imrisek M, Boboc A, Lackner K, Fernandes A, Havlicek J, Giacomelli L, Vlainic M, Nocente M, Kruezi U, Team C, Team T, Team A U, Team E M and Contributors J 2018 Nuclear Fusion 58016014 ISSN 0029-5515 URL http://stacks.iop.org/0029-5515/58/i=1/a=016014

[32] Finken K H, Watkins J G, Rusbüldt D, Corbett W J, Dippel K H, Goebel D M and Moyer R A 1990 Nuclear Fusion 30 859-870 ISSN 0029-5515 URL http://stacks.iop.org/0029-5515/30/i= $5 / \mathrm{a}=005$

[33] Kudyakov T, Jochmann A, Zeil K, Kraft S, Finken K H, Schramm U and Willi O 2009 Review of Scientific Instruments 80076106 ISSN 0034-6748 URL http://aip.scitation.org/doi/10.1063/1. 3170508

[34] Wongrach K, Finken K, Abdullaev S, Willi O, Zeng L and Xu Y 2015 Nuclear Fusion 55053008 ISSN 0029-5515 URL http://stacks.iop.org/0029-5515/55/i=5/a=053008

[35] Yoshino R, Tokuda S and Kawano Y 1999 Nuclear Fusion 39 151-161 ISSN 0029-5515 URL http://iopscience.iop.org/0029-5515/39/2/302

[36] Fredrickson E, Bell M, Taylor G and Medley S 2015 Nuclear Fusion 55013006 URL http: //stacks.iop.org/0029-5515/55/i=1/a=013006

[37] Cooper C M, Pace D C, Commaux N, Eidietis N W, Hollmann E M and Shiraki D 2016 Review of Scientific Instruments 87 11E602 URL https://aip.scitation.org/doi/abs/10.1063/1.4961288

[38] Pace D C, Cooper C M, Taussig D, Eidietis N W, Hollmann E M, Riso V, Van Zeeland M A and Watkins M 2016 Review of Scientific Instruments 87043507 ISSN 0034-6748 URL http://scitation.aip.org/content/aip/journal/rsi/87/4/10.1063/1.4945566

[39] Rigamonti D, Muraro A, Nocente M, Perseo V, Boltruczyk G, Fernandes A, Figueiredo J, Giacomelli L, Gorini G, Gosk M, Kiptily V, Korolczuk S, Mianowski S, Murari A, Pereira R C, Cippo E P, Zychor I, Tardocchi M and Contributors J 2016 Review of Scientific Instruments 87 11E717 ISSN 0034-6748 URL http://aip.scitation.org/doi/10.1063/1.4961060

[40] Nocente M, Rigamonti D, Perseo V, Tardocchi M, Boltruczyk G, Broslawski A, Cremona A, Croci 
G, Gosk M, Kiptily V, Korolczuk S, Mazzocco M, Muraro A, Strano E, Zychor I, Gorini G and Contributors J 2016 Review of Scientific Instruments 87 11E714 ISSN 0034-6748 URL http://aip.scitation.org/doi/10.1063/1.4961073

[41] Dal Molin A, Martinelli L, Nocente M, Rigamonti D, Abba A, Giacomelli L, Gorini G, Lvovskiy A, Muraro A, Tardocchi M and Contributors J 2018 Review of Scientific Instruments $8910 \mathrm{I} 134$ ISSN 0034-6748 URL http://aip.scitation.org/doi/10.1063/1.5038803

[42] Lvovskiy A, Nocente M, Dal Molin A, Eidietis N W, Giacomelli L, Martinelli L, Paz-Soldan C, Rigamonti D, Tardocchi M and Taussig D 2019 Upgrade of the Gamma Ray Imager on DIII-D (for submission to Review of Scientific Instruments)

[43] Austin M E and Lohr J 2003 Review of Scientific Instruments 74 1457-1459 ISSN 0034-6748 URL http://aip.scitation.org/doi/10.1063/1.1530387

[44] Dreicer H 1959 Physical Review 115 URL https://doi.org/10.1103/PhysRev.115.238

[45] Rosenbluth M N and Putvinski S V 1997 Nuclear Fusion 37 1355-1362 URL https://doi.org/10. 1088/0029-5515/37/10/I03

[46] Connor J W and Hastie R J 1975 Nuclear Fusion 15 415-424 ISSN 0029-5515 URL http: //stacks.iop.org/0029-5515/15/i=3/a=007

[47] Parks P B, Rosenbluth M N and Putvinski S V 1999 Physics of Plasmas 62523 ISSN 1070664X URL http://aip.scitation.org/doi/10.1063/1.873524

[48] Stahl A, Landreman M, Papp G, Hollmann E and Fulop T 2013 Physics of Plasmas 20093302 ISSN 1070-664X URL http://aip.scitation.org/doi/10.1063/1.4821823

[49] Decker J, Hirvijoki E, Embreus O, Peysson Y, Stahl A, Pusztai I and Fülöp T 2016 Plasma Physics and Controlled Fusion 58025016 ISSN 0741-3335 URL http://stacks.iop.org/0741-3335/58/i= $2 / \mathrm{a}=025016$

[50] Fülöp T, Embréus O, Stahl A, Newton S and Pusztai I 2016 Kinetic Modelling of Runaways in Fusion Plasmas Preprint: 2016 IAEA Fusion Energy Conference FEC, Kyoto, Japan, [TH/P4-1] URL https://nucleus.iaea.org/sites/fusionportal/SharedDocuments/ FEC2016/fec2016-preprints/preprint0465.pdf

[51] Guan X, Qin H and Fisch N J 2010 Physics of Plasmas 17092502 ISSN 1070-664X URL http://aip.scitation.org/doi/10.1063/1.3476268

[52] Russo A 1991 Nuclear Fusion 31 117-126 ISSN 0029-5515 URL http://stacks.iop.org/0029-5515/ $31 / \mathrm{i}=1 / \mathrm{a}=011$

[53] Liu C, Hirvijoki E, Fu G Y, Brennan D P, Bhattacharjee A and Paz-Soldan C 2018 Physical Review Letters 120265001 ISSN 0031-9007 URL https://link.aps.org/doi/10.1103/PhysRevLett.120. 265001

[54] Paz-Soldan C, Eidietis N W, Granetz R, Hollmann E M, Moyer R A, Wesley J C, Zhang J, Austin M E, Crocker N A, Wingen A and Zhu Y 2014 Physics of Plasmas 21022514 ISSN 1070-664X URL http://scitation.aip.org/content/aip/journal/pop/21/2/10.1063/1.4866912

[55] Aleynikov P and Breizman B N 2015 Physical Review Letters 114155001 ISSN 0031-9007 URL http://link.aps.org/doi/10.1103/PhysRevLett.114.155001

[56] Eriksson L G, Helander P, Andersson F, Anderson D and Lisak M 2004 Physical Review Letters 92205004 ISSN 0031-9007 URL http://link.aps.org/doi/10.1103/PhysRevLett.92.205004

[57] Smith H, Helander P, Eriksson L G, Anderson D, Lisak M and Andersson F 2006 Physics of Plasmas 13102502 ISSN 1070664X URL http://scitation.aip.org/content/aip/journal/pop/13/ 10/10.1063/1.2358110

[58] Martín-Solís J, Loarte A and Lehnen M 2017 Nuclear Fusion 57066025 ISSN 0029-5515 URL http://stacks.iop.org/0029-5515/57/i=6/a =066025

[59] Smith H, Fehér T, Fülöp T, Gál K and Verwichte E 2009 Plasma Physics and Controlled Fusion 51124008 URL http://iopscience.iop.org/0741-3335/51/12/124008

[60] Matsuyama A, Aiba N and Yagi M 2017 Nuclear Fusion 57066038 ISSN 0029-5515 URL https://doi.org/10.1088/1741-4326/aa6867

[61] Aleynikova K, Huijsmans G T A and Aleynikov P 2016 Plasma Physics Reports 42 486-494 ISSN 
1063-780X URL http://link.springer.com/10.1134/S1063780X16050019

[62] Bandaru V, Artola F J, Hoelzl M, Papp G, Aleynikov P and Huijsmans G T A Non-linear Interaction of Runaway Electrons with Resistive MHD Modes in an ITER VDE Preprint: 2018 IAEA Fusion Energy Conference, Ahmedabad, India [TH/P8-8] URL https://conferences.iaea. org/indico/event/151/papers/6421/files/4327-preprint.pdf

[63] Cai H and Fu G 2015 Nuclear Fusion 55022001 ISSN 0029-5515 URL http://stacks.iop.org/ $0029-5515 / 55 / \mathrm{i}=2 / \mathrm{a}=022001$

[64] Gill R D, Alper B, Edwards A W, Ingesson L C, Johnson M F and Ward D J 2000 Nuclear Fusion 40 163-174 ISSN 0029-5515 URL http://stacks.iop.org/0029-5515/40/i=2/a=302

[65] Loarte A, Riccardo V, Martin-Solís J, Paley J, Huber A and Lehnen M 2011 Nuclear Fusion 51 073004 ISSN 0029-5515 URL http://stacks.iop.org/0029-5515/51/i=7/a=073004

[66] Paz-Soldan C, Eidietis N W, Liu Y Q Q, Shiraki D, Boozer A H, Hollmann E M, Kim C C and Lvovskiy A 2019 Plasma Physics and Controlled Fusion 61054001 ISSN 0741-3335 URL http://iopscience.iop.org/article/10.1088/1361-6587/aafd15

[67] Humphreys D A and Kellman A G 1999 Physics of Plasmas 62742 ISSN 1070664X URL http://scitation.aip.org/content/aip/journal/pop/6/7/10.1063/1.873231

[68] Eidietis N W and Humphreys D A 2011 Nuclear Fusion 51 ISSN 00295515 URL https://iopscience. iop.org/article/10.1088/0029-5515/51/7/073034

[69] Lao L, St John H, Stambaugh R, Kellman A and Pfeiffer W 1985 Nuclear Fusion 25 1611-1622 ISSN 0029-5515 URL http://stacks.iop.org/0029-5515/25/i=11/a=007

[70] National Institute of Standarts and Technology URL https://physics.nist.gov/PhysRefData/ $\mathrm{Xcom} / \mathrm{html} / \mathrm{xcom} 1 . \mathrm{html}$

[71] Goodall D 1982 Journal of Nuclear Materials 111-112 11-22 ISSN 0022-3115

[72] McDevitt C J, Guo Z and Tang X Z 2019 Plasma Physics and Controlled Fusion 61024004 ISSN 0741-3335 URL http://stacks.iop.org/0741-3335/61/i=2/a=024004

[73] Heidbrink W W 1986 Review of Scientific Instruments 57 1769-1770 ISSN 0034-6748 URL http://aip.scitation.org/doi/10.1063/1.1139176

[74] Colchin R J, Hillis D L, Maingi R, Klepper C C and Brooks N H 2003 Review of Scientific Instruments 74 2068-2070 ISSN 0034-6748 URL http://aip.scitation.org/doi/10.1063/1.1537038

[75] Brooks N H, Colchin R J, Fehling D T, Hillis D L, Mu Y and Unterberg E 2008 Review of Scientific Instruments 79 10F330 ISSN 0034-6748 URL http://aip.scitation.org/doi/10.1063/1.2957777

[76] Lai W N, Chapman S C and Dendy R O 2013 Physics of Plasmas 20 ISSN 1070664X

[77] Vlasenkov V, Leonov V, Merezhkin V and Mukhovatov V 1973 Nuclear Fusion 13509 ISSN 0029-5515 URL http://iopscience.iop.org/article/10.1088/0029-5515/13/4/003

[78] Zhou R J, Hu L Q, Li E Z, Xu M, Zhong G Q, Xu L Q, Lin S Y, Zhang J Z and Team t E 2013 Plasma Physics and Controlled Fusion 55055006 ISSN 0741-3335 URL http://stacks.iop.org/ $0741-3335 / 55 / \mathrm{i}=5 / \mathrm{a}=055006$

[79] Causa F, Buratti P and Team F 2019 Nuclear Fusion 59046013 ISSN 0029-5515 URL http: //stacks.iop.org/0029-5515/59/i=4/a=046013

[80] Spong D A, Heidbrink W W, Paz-Soldan C, Du X D, Thome K E, Zeeland M A V, Collins C, Lvovskiy A, Moyer R A, Austin M E, Brennan D P, Liu C, Jaeger E F and Lau C 2018 Physical Review Letters 120155002 URL https://journals.aps.org/prl/abstract/10.1103/PhysRevLett. 120.155002

[81] Heidbrink W W, Paz-Soldan C, Spong D A, Du X D, Thome K E, Austin M E, Lvovskiy A, Moyer R A, Pinsker R I and Zeeland M A V 2019 Plasma Physics and Controlled Fusion 61014007 ISSN 0741-3335 URL http://stacks.iop.org/0741-3335/61/i=1/a=014007

[82] Lvovskiy A, Paz-Soldan C, Eidietis N W, Molin A D, Du X D, Giacomelli L, Herfindal J L, Hollmann E M, Martinelli L, Moyer R A, Nocente M, Rigamonti D, Shiraki D, Tardocchi M and Thome K E 2018 Plasma Physics and Controlled Fusion 60124003 ISSN 0741-3335 URL http://stacks.iop.org/0741-3335/60/i=12/a=124003

[83] Liu Y Q, Bondeson A, Fransson C M, Lennartson B and Breitholtz C 2000 Physics of Plasmas 7 
3681-3690 ISSN 1070-664X URL http://aip.scitation.org/doi/10.1063/1.1287744

[84] Liu Y, Parks P, Paz-Soldan C, Kim C and Lao L 2019 Nuclear Fusion 59126021 ISSN 0029-5515 URL https://iopscience.iop.org/article/10.1088/1741-4326/ab3f87

[85] Liu C, Shi L, Hirvijoki E, Brennan D P, Bhattacharjee A, Paz-Soldan C and Austin M E 2018 Nuclear Fusion 58096030 ISSN 0029-5515 URL http://stacks.iop.org/0029-5515/58/i=9/a= 096030

[86] Knoll G F 1999 Radiation detection and measurements 3rd ed (John Wiley \& Sons, Inc.) 